\title{
Flavor violating Higgs decays
}

\author{
Roni Harnik $^{a}$ Joachim Kopp ${ }^{a, b}$ Jure Zupan ${ }^{c}$ \\ ${ }^{a}$ Fermilab, \\ P.O. Box 500, Batavia, IL 60510, U.S.A. \\ ${ }^{b}$ Max Planck Institute for Nuclear Physics, \\ PO Box 103980, 69029 Heidelberg, Germany \\ ${ }^{c}$ Department of Physics, University of Cincinnati, \\ Cincinnati, Ohio 45221, U.S.A. \\ E-mail: roni@fnal.gov, jkopp@fnal.gov, zupanje@ucmail.uc.edu
}

ABSTRACT: We study a class of nonstandard interactions of the newly discovered $125 \mathrm{GeV}$ Higgs-like resonance that are especially interesting probes of new physics: flavor violating Higgs couplings to leptons and quarks. These interaction can arise in many frameworks of new physics at the electroweak scale such as two Higgs doublet models, extra dimensions, or models of compositeness. We rederive constraints on flavor violating Higgs couplings using data on rare decays, electric and magnetic dipole moments, and meson oscillations. We confirm that flavor violating Higgs boson decays to leptons can be sizeable with, e.g., $h \rightarrow \tau \mu$ and $h \rightarrow \tau e$ branching ratios of $\mathcal{O}(10 \%)$ perfectly allowed by low energy constraints. We estimate the current LHC limits on $h \rightarrow \tau \mu$ and $h \rightarrow \tau e$ decays by recasting existing searches for the SM Higgs in the $\tau \tau$ channel and find that these bounds are already stronger than those from rare tau decays. We also show that these limits can be improved significantly with dedicated searches and we outline a possible search strategy. Flavor violating Higgs decays therefore present an opportunity for discovery of new physics which in some cases may be easier to access experimentally than flavor conserving deviations from the Standard Model Higgs framework.

KeYwords: Higgs Physics, Rare Decays, Beyond Standard Model

ARXIV EPRINT: 1209.1397 


\section{Contents}

1 Introduction 1

2 The framework $\quad 3$

3 Leptonic flavor violating Higgs decays $\quad \mathbf{5}$

3.1 Constraints from $\tau \rightarrow \mu \gamma, \tau \rightarrow e \gamma$ and $\mu \rightarrow e \gamma \quad 6$

3.2 Constraints from $\tau \rightarrow 3 \mu, \tau \rightarrow 3 e, \mu \rightarrow 3 e \quad 8$

3.3 Constraints from muonium-antimuonium oscillations 9

$\begin{array}{ll}3.4 \text { Constraints from magnetic dipole moments } & 10\end{array}$

$\begin{array}{ll}3.5 \text { Constraints from electric dipole moments } & 11\end{array}$

3.6 Constraints from $\mu \rightarrow e$ conversion in nuclei 11

$\begin{array}{lll}3.7 & \text { LEP constraints } & 12\end{array}$

3.8 Allowed branching ratios for lepton flavor violating Higgs decays 13

4 Hadronic flavor violating decays of the Higgs $\quad 13$

$\begin{array}{lll}4.1 & \text { Flavor violating Higgs decays into light quarks } & 13\end{array}$

$\begin{array}{lll}4.2 & \text { Higgs decays through off-shell top and top decays to Higgs } & 16\end{array}$

5 Searching for flavor violating Higgs decays at the LHC 18

$\begin{array}{lll}5.1 & \text { Extracting a bound on Higgs decays to } \tau \mu \text { and } \tau e & 18\end{array}$

5.2 Comparison of $h \rightarrow \tau \mu$ to $h \rightarrow \tau \tau \quad 21$

$\begin{array}{lll}5.3 & \text { A dedicated } h \rightarrow \tau \mu \text { analysis } & 22\end{array}$

6 Conclusions $\quad 24$

A Further details on leptonic FCNCs $\quad \mathbf{2 5}$

A.1 One loop expressions for $\tau \rightarrow \mu \gamma, \tau \rightarrow e \gamma, \mu \rightarrow e \gamma \quad 26$

A.2 Two loop expressions for $\tau \rightarrow \mu \gamma, \tau \rightarrow e \gamma$ and $\mu \rightarrow e \gamma$

A.3 Details on $\mu \rightarrow e$ conversion bounds 28

\section{Introduction}

Both ATLAS and CMS have recently announced the discovery of a Higgs-like resonance with a mass of $m_{h} \simeq 125 \mathrm{GeV}$ [1-4], further supported by combined Tevatron data [5]. An interesting question is whether the properties of this resonance are consistent with the Standard Model (SM) Higgs boson. Deviations from the SM predictions could point to the existence of a secondary mechanism of electroweak symmetry breaking or to other types of new physics not too far above the electroweak scale. While there is a large ongoing experimental effort to measure precisely the decay rates into the channels that dominate 
for the SM Higgs, it is equally important to search for Higgs decays into channels that are subdominant or absent in the SM. For instance, since the couplings of the Higgs boson to quarks of the first two generations and to leptons are suppressed by small Yukawa couplings in the SM, new physics contributions can easily dominate over the SM predictions. Another possibility, and the main topic of this paper, is flavor violating (FV) Higgs decays, for instance into $\tau \mu$ or $\mu e$ final states. The study of FV couplings of the Higgs boson has a long history [6-26]. In this paper, we refine the indirect bounds on the FV couplings. Most importantly, we discuss in detail possible search strategies for FV Higgs decays at the LHC and derive for the first time limits from LHC data.

As pointed out in the previous literature, and confirmed by the present analysis, the indirect constraints on many FV Higgs decays are rather weak. In particular, the branching ratios for $h \rightarrow \tau \mu$ and $h \rightarrow \tau e$ can reach up to 10\% [24]. In fact, for $h \rightarrow \tau \mu$ and $h \rightarrow \tau e$ already now, ${ }^{1}$ without targeted searches, the LHC is placing limits that are comparable to or even stronger than those from rare $\tau$ decays. As we shall see later, re-casting a $h \rightarrow \tau \tau$ analysis with $4.7 \mathrm{fb}^{-1}$ of $7 \mathrm{TeV}$ ATLAS data [27] gives a bound on the branching fraction of the Higgs into $\tau \mu$ or $\tau e$ around $10 \%$. We will also demonstrate that dedicated searches can be much more sensitive.

These decays could thus give a striking signature of new physics at the LHC, and we strongly encourage our experimental colleagues to include them in their searches. Another experimentally interesting set of decay channels are flavor conserving decays to the first two generations, e.g., $h \rightarrow \mu^{+} \mu^{-}$, on which we will comment further below. We emphasize that large deviations from the SM do not require very exotic flavor structures. A branching ratio for $h \rightarrow \tau \mu$ comparable to the one for $h \rightarrow \tau \tau$, or a $h \rightarrow \mu^{+} \mu^{-}$branching ratio a few times larger than in the SM can arise in many models of flavor (for instance in models with continuous and/or discrete flavor symmetries [28], or in Randall-Sundrum models [29]) as long as there is new physics at the electroweak scale and not just the SM. The lepton flavor violating decay $h \rightarrow \tau \mu$ has been studied in [11], and it was found that the branching ratio for this decay can be up to $10 \%$ in certain Two Higgs Doublet Models (2HDMs).

In fact, there may already be experimental hints that the Higgs couplings to fermions may not be SM-like. For instance, the BaBar collaboration recently announced a $3.4 \sigma$ indication of flavor universality violation in $b \rightarrow c \tau \nu$ transitions [30], which can be explained for instance by an extended Higgs sector with nontrivial flavor structure [31].

The paper is organized as follows. In section 2 we introduce the theoretical framework we will use to parameterize the flavor violating decays of the Higgs. In section 3 we derive bounds on flavor violating Higgs couplings to leptons and translate these bounds into limits on the Higgs decay branching fractions to the various flavor violating final states. In section 4 we do the same for flavor violating couplings to quarks. We shall see that decays of the Higgs to $\tau \mu$ and to $\tau e$ with sizeable branching fractions are allowed, and that also flavor violating couplings of the Higgs to top quarks are only weakly constrained. Motivated by this we turn to the LHC in section 5 and estimate the current bounds on

\footnotetext{
${ }^{1}$ By $h \rightarrow \tau \mu$ we always mean the sum of $h \rightarrow \tau^{+} \mu^{-}$and $h \rightarrow \tau^{-} \mu^{+}$and similarly for the other decay modes.
} 
Higgs decays to $\tau \mu$ and $\tau e$ using data from an existing $h \rightarrow \tau \tau$ search. We also discuss a strategy for a dedicated $h \rightarrow \tau \mu$ search and comment on differences with the SM $h \rightarrow \tau \tau$ searches. We will see that the LHC can make significant further progress in probing the Higgs' flavor violating parameters space with existing data. We conclude in section 6 . In the appendices, we give more details on the calculation of constraints from low-energy observables.

\section{The framework}

After electroweak symmetry breaking (EWSB) the fermionic mass terms and the couplings of the Higgs boson to fermion pairs in the mass basis are in general

$$
\mathcal{L}_{Y}=-m_{i} \bar{f}_{L}^{i} f_{R}^{i}-Y_{i j}\left(\bar{f}_{L}^{i} f_{R}^{j}\right) h+\text { h.c. }+\cdots,
$$

where ellipses denote nonrenormalizable couplings involving more than one Higgs field operator. In our notation, $f_{L}=q_{L}, \ell_{L}$ are $\mathrm{SU}(2)_{L}$ doublets, $f_{R}=u_{R}, d_{R}, \nu_{R}, \ell_{R}$ the weak singlets, and indices run over generations and fermion flavors (quarks and leptons) with summation implicitly understood. In the SM the Higgs couplings are diagonal, $Y_{i j}=$ $\left(m_{i} / v\right) \delta_{i j}$, but in general NP models the structure of the $Y_{i j}$ can be very different. Note that we use the normalization $v=246 \mathrm{GeV}$ here. The goal of the paper is to set bounds on $Y_{i j}$ and identify interesting channels for Higgs decays at the LHC. Throughout we will assume that the Higgs is the only additional degree of freedom with mass $\mathcal{O}(100 \mathrm{GeV})$ and that the $Y_{i j}$ 's are the only source of flavor violation. These assumptions are not necessarily valid in general, but will be a good approximation in many important classes of new physics frameworks. Let us now show how $Y_{i j} \neq\left(m_{i} / v\right) \delta_{i j}$ can arise in two qualitatively different categories of NP models.

A single Higgs theory. Let us first explore the possibility that the Higgs is the only field that causes EWSB (see also [10, 15, 19, 23, 32-34]). For simplicity let us also assume that at energies below $\sim 200 \mathrm{GeV}$ the spectrum consists solely of the SM particles: three generations of quarks and leptons, the SM gauge bosons and the Higgs at $125 \mathrm{GeV}$. Additional heavy fields (e.g. scalar or fermionic partners which address the hierarchy problem) can be integrated out, so that we can work in effective field theory (EFT) - the effective Standard Model. In addition to the SM Lagrangian

$$
\mathcal{L}_{S M}=\bar{f}_{L}^{j} i \not D f_{L}^{j}+\bar{f}_{R}^{j} i \not D f_{R}^{j}-\left[\lambda_{i j}\left(\bar{f}_{L}^{i} f_{R}^{j}\right) H+h . c .\right]+D_{\mu} H^{\dagger} D^{\mu} H-\lambda_{H}\left(H^{\dagger} H-\frac{v^{2}}{2}\right)^{2},
$$

there are then also higher dimensional terms due to the heavy degrees of freedom that were integrated out:

$$
\Delta \mathcal{L}_{Y}=-\frac{\lambda_{i j}^{\prime}}{\Lambda^{2}}\left(\bar{f}_{L}^{i} f_{R}^{j}\right) H\left(H^{\dagger} H\right)+\text { h.c. }+\cdots
$$

Here we have written out explicitly only the terms that modify the Yukawa interactions. We can truncate the expansion after the terms of dimension 6 , since these already suffice to 
completely decouple the values of the fermion masses from the values of fermion couplings to the Higgs boson. Additional dimension 6 operators involving derivatives include

$$
\Delta \mathcal{L}_{D}=\frac{\lambda_{L}^{i j}}{\Lambda^{2}}\left(\bar{f}_{L}^{i} \gamma^{\mu} f_{L}^{j}\right)\left(H^{\dagger} i \overleftrightarrow{D_{\mu}} H\right)+\frac{\lambda_{R}^{i j}}{\Lambda^{2}}\left(\bar{f}_{R}^{i} \gamma^{\mu} f_{R}^{j}\right)\left(H^{\dagger} i \overleftrightarrow{D_{\mu}} H\right)+\cdots
$$

where $\left(H^{\dagger} i \overleftrightarrow{D_{\mu}} H\right) \equiv H^{\dagger} i D_{\mu} H-\left(i D_{\mu} H^{\dagger}\right) H$. The couplings $\lambda_{i j}^{\prime}$ are complex in general, while the $\lambda_{L, R}^{i j}$ are real. The derivative couplings do not give rise to fermion-fermion-Higgs couplings after EWSB and are irrelevant for our analysis. In eq. (2.4) there are in principle also terms of the form $\left(\bar{f}_{L, R}^{i} i \not D f_{L . R}^{j}\right) H^{\dagger} H$, which, however, can be shown to be equivalent to (2.3) by using equations of motion.

After electroweak symmetry breaking (EWSB) and diagonalization of the mass matrices, one obtains the Yukawa Lagrangian in eq. (2.1), with

$$
\sqrt{2} m=V_{L}\left[\lambda+\frac{v^{2}}{2 \Lambda^{2}} \lambda^{\prime}\right] V_{R}^{\dagger} v, \quad \sqrt{2} Y=V_{L}\left[\lambda+3 \frac{v^{2}}{2 \Lambda^{2}} \lambda^{\prime}\right] V_{R}^{\dagger},
$$

where the unitary matrices $V_{L}, V_{R}$ are those which diagonalize the mass matrix, and $v=$ $246 \mathrm{GeV}$. In the mass basis we can write

$$
Y_{i j}=\frac{m_{i}}{v} \delta_{i j}+\frac{v^{2}}{\sqrt{2} \Lambda^{2}} \hat{\lambda}_{i j}
$$

where $\hat{\lambda}=V_{L} \lambda^{\prime} V_{R}$. In the limit $\Lambda \rightarrow \infty$ one obtains the SM, where the Yukawa matrix $Y$ is diagonal, $Y v=m$. For $\Lambda$ of the order of the electroweak scale, on the other hand, the mass matrix and the couplings of the Higgs to fermions can be very different as $\hat{\lambda}$ is in principle an arbitrary non-diagonal matrix.

Taking the off diagonal Yukawa couplings nonzero can come with a theoretical price. Consider, for instance, a two flavor mass matrix involving $\tau$ and $\mu$. If the off-diagonal entries are very large the mass spectrum is generically not hierarchical. A hierarchical spectrum would require a delicate cancellation among the various terms in eq. (2.5). Tuning is avoided if [35]

$$
\left|Y_{\tau \mu} Y_{\mu \tau}\right| \lesssim \frac{m_{\mu} m_{\tau}}{v^{2}}
$$

with similar conditions for the other off diagonal elements. Even though we will keep this condition in the back of our minds, we will not restrict the parameter space to fulfill it.

Models with several sources of EWSB. Let us now discuss the case where the Higgs at $125 \mathrm{GeV}$ is not the only scalar that breaks electroweak symmetry. The modification of the above discussion is straightforward. The additional sources of EWSB are assumed to be heavy and can thus still be integrated out. Their EWSB effects can be described by a spurion $\chi$ that formally transforms under electroweak global symmetry and then obtains a vacuum expectation value (vev), which breaks the electroweak symmetry. If $\chi$ has the quantum numbers $(2,1 / 2)$ under $\mathrm{SU}(2)_{L} \times \mathrm{U}(1)_{Y}$ it can contribute to quark and lepton masses. $^{2}$ This allows the Yukawa interactions $Y$ of the $125 \mathrm{GeV}$ Higgs to be misaligned with respect to the fermion mass matrix $m$ in eq. (2.1).

\footnotetext{
${ }^{2} \mathrm{~A}$ spurion which transforms as a triplet can also contribute to Majorana masses for neutrinos.
} 
The simplest example for a full theory of this class is a type III two Higgs doublet model (2HDM) where both Higgses obtain a vev and couple to fermions. In the full theory both of the scalars then have a Lagrangian of the form (2.1)

$$
\mathcal{L}_{Y}=-m_{i} \bar{f}_{L}^{i} f_{R}^{i}-Y_{i j}^{a}\left(\bar{f}_{L}^{i} f_{R}^{j}\right) h^{a}+\text { h.c. }+\cdots,
$$

where the index $a$ runs over all the scalars (with $Y_{i j}^{a}$ imaginary for pseudoscalars), and $m_{i}$ receives contributions from both vevs. In addition there is also a scalar potential which mixes the two Higgses. Diagonalizing the Higgs mass matrix then also changes $Y_{i j}^{a}$, but removes the Higgs mixing. For our purposes it is simplest to work in the Higgs mass basis. All the results for a single Higgs are then trivially modified, replacing our final expressions below by a sum over several Higgses. For a large mass gap, where only one Higgs is light, the contributions from the heavier Higgs are power suppressed, unless its flavor violating Yukawa couplings are parametrically larger than those of the light Higgs. The contributions from the heavy Higgs correspond to the higher dimensional operators discussed in the previous paragraph. This example can be trivially generalized to models with many Higgs doublets.

We next derive constraints on flavor violating Higgs couplings and work out the allowed branching fractions for flavor violation Higgs decays. In placing the bounds we will neglect the FV contributions of the remaining states in the full theory. Our bounds thus apply barring cancellations with these other terms.

\section{Leptonic flavor violating Higgs decays}

The FV decays $h \rightarrow e \mu, e \tau, \mu \tau$ arise at tree level from the assumed flavor violating Yukawa interactions, eq. (2.1), where the relevant terms are explicitly

$$
\mathcal{L}_{Y} \supset-Y_{e \mu} \bar{e}_{L} \mu_{R} h-Y_{\mu e} \bar{\mu}_{L} e_{R} h-Y_{e \tau} \bar{e}_{L} \tau_{R} h-Y_{\tau e} \bar{\tau}_{L} e_{R} h-Y_{\mu \tau} \bar{\mu}_{L} \tau_{R} h-Y_{\tau \mu} \bar{\tau}_{L} \mu_{R} h+h . c . .
$$

The bounds on the FV Yukawa couplings are collected in table 1, where for simplicity of presentation the flavor diagonal muon and tau Yukawa couplings,

$$
\mathcal{L}_{Y} \supset-Y_{\mu \mu} \bar{\mu}_{L} \mu_{R} h-Y_{\tau \tau} \bar{\tau}_{L} \tau_{R} h+\text { h.c. },
$$

were set equal to their respective $\mathrm{SM}$ values $\left(Y_{\mu \mu}\right)_{\mathrm{SM}}=m_{\mu} / v,\left(Y_{\tau \tau}\right)_{\mathrm{SM}}=m_{\tau} / v$. Similar bounds on FV Higgs couplings to quarks are collected in table 2. Similar constraints on flavor violating Higgs decays have been present recently also in [24]. While our results agree qualitatively with previous ones, small numerical differences are expected because we avoid some of the approximations made by previous authors. We also consider some constraining processes not discussed before.

We first give more details on how the bounds in tables 1 and 2 were obtained and then move on to predictions for the allowed sizes of the FV Higgs decays. 


\begin{tabular}{|lcc|}
\hline Channel & Coupling & Bound \\
\hline$\mu \rightarrow e \gamma$ & $\sqrt{\left|Y_{\mu e}\right|^{2}+\left|Y_{e \mu}\right|^{2}}$ & $<3.6 \times 10^{-6}$ \\
$\mu \rightarrow 3 e$ & $\sqrt{\left|Y_{\mu e}\right|^{2}+\left|Y_{e \mu}\right|^{2}}$ & $\lesssim 3.1 \times 10^{-5}$ \\
electron $g-2$ & $\operatorname{Re}\left(Y_{e \mu} Y_{\mu e}\right)$ & $-0.019 \ldots 0.026$ \\
electron EDM & $\left|\operatorname{Im}\left(Y_{e \mu} Y_{\mu e}\right)\right|$ & $<9.8 \times 10^{-8}$ \\
$\mu \rightarrow e$ conversion & $\sqrt{\left|Y_{\mu e}\right|^{2}+\left|Y_{e \mu}\right|^{2}}$ & $<4.6 \times 10^{-5}$ \\
$M-\bar{M}$ oscillations & $\left|Y_{\mu e}+Y_{e \mu}^{*}\right|$ & $<0.079$ \\
\hline$\tau \rightarrow e \gamma$ & $\sqrt{\left|Y_{\tau e}\right|^{2}+\left|Y_{e \tau}\right|^{2}}$ & $<0.014$ \\
$\tau \rightarrow 3 e$ & $\sqrt{\left|Y_{\tau e}\right|^{2}+\left|Y_{e \tau}\right|^{2}}$ & $\lesssim 0.12$ \\
electron $g-2$ & $\operatorname{Re}\left(Y_{e \tau} Y_{\tau e}\right)$ & {$[-2.1 \ldots 2.9] \times 10^{-3}$} \\
electron EDM & $\left|\operatorname{Im}\left(Y_{e \tau} Y_{\tau e}\right)\right|$ & $<1.1 \times 10^{-8}$ \\
\hline$\tau \rightarrow \mu \gamma$ & $\sqrt{\left|Y_{\tau \mu}\right|^{2}+\left|Y_{\mu \tau}\right|^{2}}$ & 0.016 \\
$\tau \rightarrow 3 \mu$ & $\sqrt{\left.\left|Y_{\tau \mu}^{2}+\right| Y_{\mu \tau}\right|^{2}}$ & $\lesssim 0.25$ \\
muon $g-2$ & $\operatorname{Re}\left(Y_{\mu \tau} Y_{\tau \mu}\right)$ & $(2.7 \pm 0.75) \times 10^{-3}$ \\
muon EDM & $\operatorname{Im}\left(Y_{\mu \tau} Y_{\tau \mu}\right)$ & $-0.8 \ldots 1.0$ \\
\hline$\mu \rightarrow e \gamma$ & $\left(\left|Y_{\tau \mu} Y_{\tau e}\right|^{2}+\left|Y_{\mu \tau} Y_{e \tau}\right|^{2}\right)^{1 / 4}$ & $<3.4 \times 10^{-4}$ \\
\hline
\end{tabular}

Table 1. Constraints on flavor violating Higgs couplings to $e, \mu, \tau$ for a Higgs mass $m_{h}=125 \mathrm{GeV}$ and assuming that the flavor diagonal Yukawa couplings equal the SM values (see text for details). For the muon magnetic dipole moment we show the value of the couplings required to explain the observed $\Delta a_{\mu}$ (if this is used only as an upper bound one has $\sqrt{\operatorname{Re}\left(Y_{\mu \tau} Y_{\tau \mu}\right)}<0.065$ at $95 \% \mathrm{CL}$ ).

\subsection{Constraints from $\boldsymbol{\tau} \rightarrow \mu \gamma, \boldsymbol{\tau} \rightarrow e \gamma$ and $\boldsymbol{\mu} \rightarrow e \gamma$}

The effective Lagrangian for the $\tau \rightarrow \mu \gamma$ decay is given by

$$
\mathcal{L}_{\text {eff }}=c_{L} Q_{L \gamma}+c_{R} Q_{R \gamma}+\text { h.c. },
$$

where the dim-5 electromagnetic penguin operators are

$$
Q_{L \gamma, R \gamma}=\frac{e}{8 \pi^{2}} m_{\tau}\left(\bar{\mu} \sigma^{\alpha \beta} P_{L, R} \tau\right) F_{\alpha \beta},
$$

with $\alpha, \beta$ the Lorentz indices and $F_{\alpha \beta}$ the electromagnetic field strength tensor. The Wilson coefficients $c_{L}$ and $c_{R}$ receive contributions from the two 1-loop diagrams shown in figure 1 (with the first one dominant), and a comparable contribution from Barr-Zee type 2-loop diagrams, see figure 12 in appendix A. The complete one loop and two loop expressions are given in appendix A.

In the approximation $Y_{\mu \mu} \ll Y_{\tau \tau}$, only the first of the one-loop diagrams in figure 1 is relevant (in addition to the 2-loop diagrams). Using also $m_{\mu} \ll m_{\tau} \ll m_{h}$ and assuming $Y_{\mu \mu}, Y_{\tau \tau}$ to be real, the expressions for the one-loop Wilson coefficients $c_{L}$ and $c_{R}$ simplify 


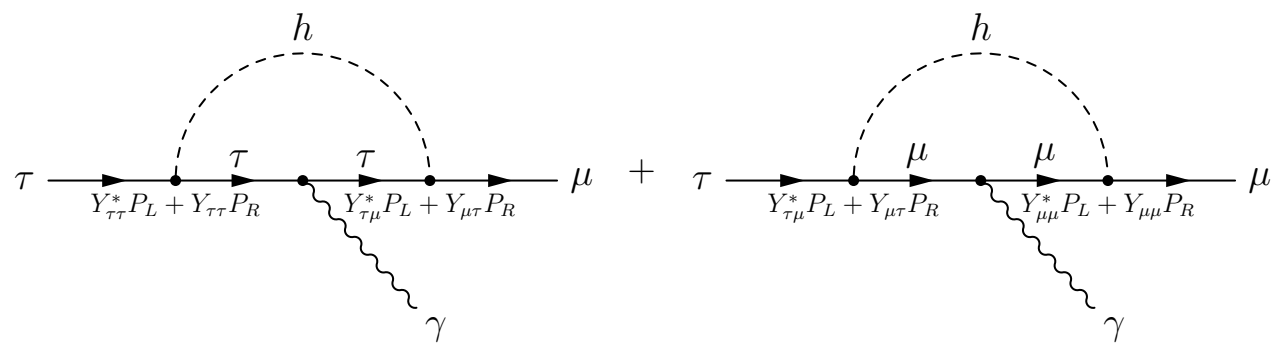

Figure 1. Diagrams contributing to the flavor violating decay $\tau \rightarrow \mu \gamma$, mediated by a Higgs boson with flavor violating Yukawa couplings.

to (this agrees with [24])

$$
c_{L}^{1 \text { loop }} \simeq \frac{1}{12 m_{h}^{2}} Y_{\tau \tau} Y_{\tau \mu}^{*}\left(-4+3 \log \frac{m_{h}^{2}}{m_{\tau}^{2}}\right), \quad c_{R}^{1 \text { loop }} \simeq \frac{1}{12 m_{h}^{2}} Y_{\mu \tau} Y_{\tau \tau}\left(-4+3 \log \frac{m_{h}^{2}}{m_{\tau}^{2}}\right)
$$

The 2-loop contributions are numerically

$$
c_{L}^{2 \text { loop }}=Y_{\tau \mu}^{*}\left(-0.082 Y_{t t}+0.11\right) \frac{1}{(125 \mathrm{GeV})^{2}}=0.055 Y_{\tau \mu}^{*} \frac{1}{(125 \mathrm{GeV})^{2}},
$$

where in the last step we used for the top Yukawa coupling $Y_{t t}=\left(Y_{t t}\right)_{S M}=\bar{m}_{t} / v=0.67$, and we have normalized the results to $m_{h}$ for easier comparison. (By $\bar{m}_{t}$, we denote the top quark mass parameter in the $\overline{\mathrm{MS}}$ renormalization scheme, $\bar{m}_{t} \simeq 164 \mathrm{GeV}$.) The analytical form of the Wilson coefficient can be found in appendix A. The same result applies to $c_{R}^{2 \text { loop }}$ with the replacement $Y_{\tau \mu}^{*} \rightarrow Y_{\mu \tau}$. The two loop contribution is dominated by two terms, the one with the top quark in the loop and of a somewhat larger $W$ contribution. They have opposite signs and thus part of $W$ contributions is cancelled. The end result has an increased sensitivity to the precise value of $Y_{t t}$. For $Y_{t t} \simeq \bar{m}_{t} / v$ the 2-loop contribution is about four times as large as the 1-loop contribution, while for other values of $Y_{t t}$ (e.g., $\left.Y_{t t} \simeq-m_{t} / v\right)$ the 2-loop contribution can be an order of magnitude larger. Note that we keep complete 2-loop expressions [36], including the finite terms, while in [20, 24] only the leading log term of the top loop contribution was kept. Numerically, this amounts to an $\mathcal{O}(1)$ difference.

In terms of the Wilson coefficients $c_{L}$ and $c_{R}$, the rate for $\tau \rightarrow \mu \gamma$ is

$$
\Gamma(\tau \rightarrow \mu \gamma)=\frac{\alpha m_{\tau}^{5}}{64 \pi^{4}}\left(\left|c_{L}\right|^{2}+\left|c_{R}\right|^{2}\right)
$$

Using a Higgs mass $m_{h}=125 \mathrm{GeV}$ and assuming $Y_{\tau \tau}=m_{\tau} / v, Y_{t t}=\bar{m}_{t} / v$, we can then translate the experimental bound $\operatorname{BR}(\tau \rightarrow \mu \gamma)<4.4 \times 10^{-8}$ [37] into a constraint $\sqrt{\left|Y_{\tau \mu}\right|^{2}+\left|Y_{\mu \tau}\right|^{2}}<1.6 \times 10^{-2}$ (see table 1). The bound is relaxed if $Y_{\tau \tau}$ and/or $Y_{t t}$ are smaller than their SM values.

The expressions for $\mu \rightarrow e \gamma$ and $\tau \rightarrow e \gamma$ are obtained in an analogous way with the obvious replacements $(\tau \rightarrow \mu, \mu \rightarrow e$ for the first and $\mu \rightarrow e$ for the second in eqs. (3.4), (3.5), (3.6), (3.7)). The experimental bound $\operatorname{BR}(\mu \rightarrow e \gamma)<2.4 \cdot 10^{-12}$ [37] 


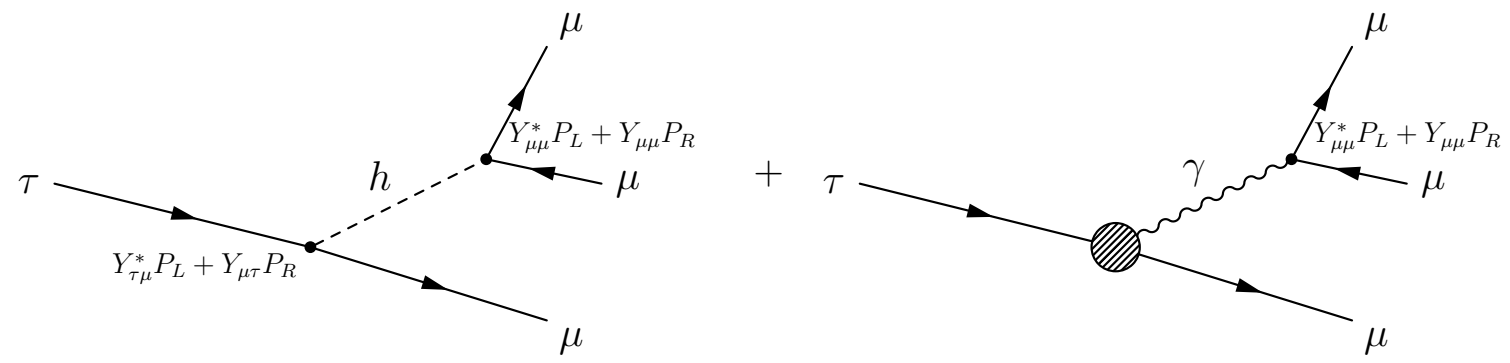

Figure 2. Diagrams leading to $\tau \rightarrow 3 \mu$ decay. The tree level Higgs exchange contribution (left) is typically subdominant compared to higher-order contributions with the topology shown on the right. The blob represents loops of the form shown in figures 1 and 12 .

then translates to $\sqrt{\left|Y_{\mu e}\right|^{2}+\left|Y_{e \mu}\right|^{2}}<3.6 \times 10^{-6}$ and $\mathrm{BR}(\tau \rightarrow e \gamma)<3.3 \times 10^{-8}$ [37] to a constraint $\sqrt{\left|Y_{\tau e}\right|^{2}+\left|Y_{e \tau}\right|^{2}}<1.4 \times 10^{-2}$ using the SM values for $Y_{\tau \tau}, Y_{\mu \mu}$, and $Y_{t t}$. The $\mu \rightarrow e \gamma$ bound is completely dominated by the two loop contribution, while for $\tau \rightarrow e \gamma$, the two loop and one loop contributions are comparable.

The decay $\mu \rightarrow e \gamma$ can also be used to place a bound on the combination $Y_{\mu \tau} Y_{\tau e}$ using the 1-loop Wilson coefficient (in agreement with [24])

$$
c_{L}^{1 \text { loop }} \simeq \frac{1}{8 m_{h}^{2}} \frac{m_{\tau}}{m_{\mu}} Y_{\mu \tau}^{*} Y_{\tau e}^{*}\left(-3+2 \log \frac{m_{h}^{2}}{m_{\tau}^{2}}\right) .
$$

As before, $c_{R}^{1 \text { loop }}$ is obtained by replacing $Y_{\mu \tau}^{*} Y_{\tau e}^{*}$ by $Y_{\tau \mu} Y_{e \tau}$. The 2-loop contribution is proportional to $Y_{\mu e}$ and $Y_{e \mu}$. Setting them to zero, one obtains a bound $\left(\left|Y_{\tau \mu} Y_{\tau e}\right|^{2}+\right.$ $\left.\left|Y_{\mu \tau} Y_{e \tau}\right|^{2}\right)^{1 / 4}<3.4 \times 10^{-4}$.

\subsection{Constraints from $\boldsymbol{\tau} \rightarrow 3 \mu, \boldsymbol{\tau} \rightarrow 3 e, \boldsymbol{\mu} \rightarrow 3 e$}

The decay $\tau \rightarrow 3 \mu$ can be generated through tree level Higgs exchange, see the diagram in figure 2 (left). However, the diagram is suppressed not only by the flavor violating Yukawa couplings $Y_{\tau \mu}$ and $Y_{\mu \tau}$, but also by the flavor-conserving coupling $Y_{\mu \mu}$. It is thus subleading compared to the higher order contributions: the 1-loop diagrams of the form shown in figure 1 and 2-loop diagrams like the ones shown in figure 12 (for $\tau \rightarrow \mu \gamma$ ). These generate $\tau \rightarrow 3 \mu$ if the outgoing gauge boson is off-shell and "decays" to a muon pair. This general topology is shown in the right part of figure 2 .

Integrating out the Higgs, the heavy gauge bosons and the top quark, these contributions match onto an effective Lagrangian. The full effective Lagrangian is similar to the one in eq. (A.13) for $\mu \rightarrow e$ conversion, but with quarks replaced by muons. Since a full evaluation of the 2-loop contributions is beyond the scope of this work, we will estimate the $\tau \rightarrow 3 \mu$ rate by including only the dimension 5 elecromagnetic dipole contributions of the form given in eq. (3.4). For $c_{L}$ and $c_{R}$, we use the same expressions as for $\tau \rightarrow \mu \gamma$, see section 3.1 and appendix A. We evaluate these expressions at $q^{2}=0$. We have checked that the neglected contributions are numerically smaller than the dipole terms at one loop. At two loops, to the best of our knowledge, a full evaluation of all potentially relevant diagrams is not available. 


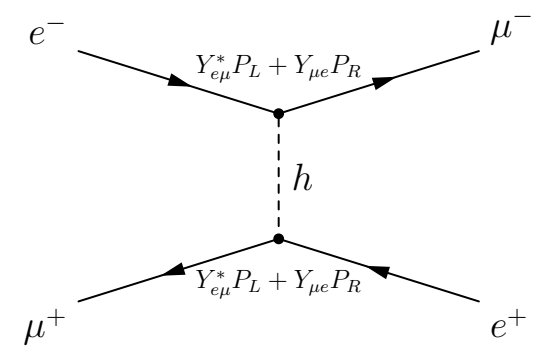

Figure 3. Diagram leading to muonium-antimuonium oscillations.

The corresponding expression for the flavor violating partial width of the $\tau$ is

$$
\Gamma(\tau \rightarrow 3 \mu) \simeq \frac{\alpha^{2} m_{\tau}^{5}}{72(2 \pi)^{5}}\left[12 \log \frac{m_{\mu}^{2}}{m_{\tau}^{2}}+29+6 \log 4\right]\left(\left|c_{L}\right|^{2}+\left|c_{R}\right|^{2}\right),
$$

where we have neglected terms additionally suppressed by the muon mass. The Wilson coefficients $c_{L}$ and $c_{R}$ are given approximately by eqs. (3.5) and (3.6), with the 2-loop contribution dominating over the 1-loop one.

The experimental bound $\operatorname{BR}(\tau \rightarrow 3 \mu)<2.1 \times 10^{-8}$ [38] translates into a constraint $\sqrt{\left.\left|Y_{\tau \mu}^{2}+\right| Y_{\mu \tau}\right|^{2}}<0.25$ for $m_{h}=125 \mathrm{GeV}$ and assuming that the diagonal Yukawa couplings $Y_{\tau \tau}, Y_{\mu \mu}$ and $Y_{t t}$ have their Standard Model values. The decay $\tau \rightarrow 3 \mu$ thus leads to a weaker limit on $Y_{\tau \mu}, Y_{\mu \tau}$ than $\tau \rightarrow \mu \gamma$, mainly because $\Gamma(\tau \rightarrow 3 \mu)$ is suppressed by an additional power of $\alpha$ compared to $\Gamma(\tau \rightarrow \mu \gamma)$.

Similarly, the constraints on $Y_{\mu e}, Y_{e \mu}, Y_{\tau e}, Y_{e \tau}$ following from the processes $\mu \rightarrow 3 e$ and $\tau \rightarrow 3 e$ are weaker than the corresponding limits from $\mu \rightarrow e \gamma$ and $\tau \rightarrow e \gamma$. The bounds in table 1 are obtained using the experimental results $\operatorname{BR}(\mu \rightarrow 3 e)<1.0 \times 10^{-12}$ [39] and $\operatorname{BR}(\tau \rightarrow 3 e)<2.7 \times 10^{-8}[40]$. We have also considered the process $\tau \rightarrow e \mu \mu$, but found that it yields a weaker limit than $\tau \rightarrow 3 e$, mainly because of the smaller phase space.

\subsection{Constraints from muonium-antimuonium oscillations}

A $\mu^{+} e^{-}$bound state (called muonium $M$ ) can oscillate into an $e^{+} \mu^{-}$bound state (antimuonium $\bar{M}$ ) through the diagram in figure 3. The time-integrated $M \rightarrow \bar{M}$ conversion probability is constrained by the MACS experiment at PSI [41] to be below $P(M \rightarrow \bar{M})<$ $8.3 \times 10^{-11} / S_{B}$, where the correction factor $S_{B} \leq 1$ accounts for the splitting of muonium states in the magnetic field of the detector. It depends on the Lorentz structure of the conversion operator and varies between $S_{B}=0.35$ for $(S \pm P) \times(S \pm P)$ operators and $S_{B}=0.9$ for $P \times P$ operators [41]. Conservatively, we use the smallest value $S_{B}=0.35$ throughout. Since we will find that $M-\bar{M}$ oscillation constraints are much weaker than those from from $\mu \rightarrow e \gamma$ and $\mu \rightarrow e$ conversion, this approximation suffices for illustrative purposes.

The theoretical prediction for the $M \rightarrow \bar{M}$ conversion rate is governed by the mixing matrix element (see, e.g., [42])

$$
\mathcal{M}_{\bar{M} M}=\left\langle\uparrow_{\mu} \downarrow_{\bar{e}}-\downarrow_{\mu} \uparrow_{\bar{e}}\left|\frac{\left[\bar{\mu}\left(Y_{e \mu}^{*} P_{L}+Y_{\mu e} P_{R}\right) e\right]\left[\bar{\mu}\left(Y_{e \mu}^{*} P_{L}+Y_{\mu e} P_{R}\right) e\right]}{2 m_{h}^{2}}\right| \uparrow_{e \downarrow_{\bar{\mu}}}-\downarrow_{e} \uparrow_{\bar{\mu}}\right\rangle,
$$




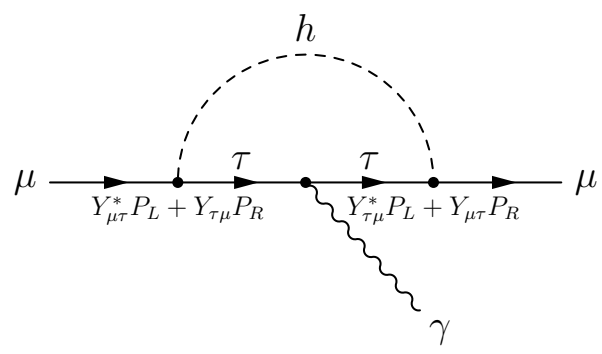

Figure 4. A diagram contributing to the anomalous magnetic moment $g-2$ of the muon through FV couplings of the Higgs to $\tau \mu$.

where $\uparrow_{X}$ and $\downarrow_{X}$ are the spin orientations of particle $X$. We can work in the nonrelativistic limit here. For a contact interaction, the spatial wave function of muonium, $\phi_{1 s}=\exp \left(-r / a_{M}\right) /\left[\pi a_{M}^{3}\right]^{1 / 2}$, only needs to be evaluated at the origin. (Here $r$ is the electron-antimuon distance and $a_{M}=\left(m_{e}+m_{\mu}\right) /\left(m_{e} m_{\mu} \alpha\right)$ is the muonium Bohr radius.) The resulting mass splitting between the two mass eigenstates of the mixed $M-\bar{M}$ system is [42],

$$
\Delta M=2\left|\mathcal{M}_{\bar{M} M}\right|=\frac{\left|Y_{\mu e}+Y_{e \mu}^{*}\right|^{2}}{2 \pi a^{3} m_{h}^{2}}
$$

and the time-integrated conversion probability is

$$
P(M \rightarrow \bar{M})=\int_{0}^{\infty} d t \Gamma_{\mu} \sin ^{2}(\Delta M t) e^{-\Gamma_{\mu} t}=\frac{2}{\Gamma_{\mu}^{2} /(\Delta M)^{2}+4} .
$$

The bound from the MACS experiment [41] then translates into $\left|Y_{\mu e}+Y_{e \mu}^{*}\right|<0.079$.

\subsection{Constraints from magnetic dipole moments}

The $\mathrm{CP}$ conserving and $\mathrm{CP}$ violating parts of the diagram in figure 4 generate magnetic and electric dipole moments of the muon, respectively. Since the experimental value of the magnetic dipole moment, $g_{\mu}-2$, is above the SM prediction at more than $3 \sigma$, also the preferred value for the flavor violating Higgs couplings will be nonzero.

The FV contribution to $(g-2)_{\mu}$ due to the $\tau$-Higgs loop in figure 4 is (neglecting terms suppressed by $m_{\mu} / m_{\tau}$ or $\left.m_{\tau} / m_{h}\right)$

$$
a_{\mu} \equiv \frac{g_{\mu}-2}{2} \simeq \frac{\operatorname{Re}\left(Y_{\mu \tau} Y_{\tau \mu}\right)}{8 \pi^{2}} \frac{m_{\mu} m_{\tau}}{2 m_{h}^{2}}\left(2 \log \frac{m_{h}^{2}}{m_{\tau}^{2}}-3\right)
$$

in agreement with [24]. The discrepancy between the measured value of $a_{\mu}$ and the one predicted by the Standard Model [38, 43],

$$
\Delta a_{\mu} \equiv a_{\mu}^{\exp }-a_{\mu}^{\mathrm{SM}}=(2.87 \pm 0.63 \pm 0.49) \times 10^{-9},
$$

could thus be explained if there are FV Higgs interactions of the size

$$
\operatorname{Re}\left(Y_{\mu \tau} Y_{\tau \mu}\right) \simeq(2.7 \pm 0.75) \times 10^{-3}
$$


(for the definition of the Yukawa couplings see eq. (2.1)). This explanation of $\Delta a_{\mu}$ requires $Y_{\mu \tau} \sim Y_{\tau \mu}$ to be a factor of a few bigger than the SM value of the diagonal Yukawa, $m_{\tau} / v$, and is in tension with limits from $\tau \rightarrow \mu \gamma .{ }^{3}$ It is in further tension with the LHC limit extracted in section 5 of this paper.

The measured $\Delta a_{\mu}$ could in principle also be explained by an enhanced flavor conserving coupling of the muon to the Higgs if $Y_{\mu \mu} \sim 0.15 \sim 280 m_{\mu} / v$. However, in this case $h \rightarrow \mu \mu$ decays would be enhanced to a level that is already ruled out by the searches at the LHC: from the search for the MSSM neutral Higgs boson one obtains a bound $\sigma(g g \rightarrow h \rightarrow \mu \mu) \lesssim 30 \times \sigma(g g \rightarrow h \rightarrow \mu \mu)_{\mathrm{SM}}$ or $Y_{\mu \mu} \lesssim 5.5 m_{\mu} / v[44]$.

\subsection{Constraints from electric dipole moments}

If the flavor violating Yukawa couplings in figure 4 are complex, the diagram shown there generates also an electric dipole moment (EDM) for the muon. The relevant term in the effective Lagrangian is

$$
\mathcal{L}_{\mathrm{EDM}}=-\frac{i}{2} d_{\mu}\left(\bar{\mu} \sigma^{\alpha \beta} \gamma^{5} \mu\right) F_{\alpha \beta}
$$

with the electric dipole moment given by (neglecting the terms suppressed by $m_{\mu} / m_{\tau}$ or $\left.m_{\tau} / m_{h}\right)$

$$
d_{\mu} \simeq-\frac{\operatorname{Im}\left(Y_{\mu \tau} Y_{\tau \mu}\right)}{16 \pi^{2}} \frac{e m_{\tau}}{2 m_{h}^{2}}\left(2 \log \frac{m_{h}^{2}}{m_{\tau}^{2}}-3\right)
$$

in agreement with [24]. The experimental constraint $-10 \times 10^{-20} e \mathrm{~cm}<d_{\mu}<8 \times$ $10^{-20} e \mathrm{~cm}$ [37] translates into the rather weak limit $-0.8 \lesssim \operatorname{Im}\left(Y_{\mu \tau} Y_{\tau \mu}\right) \lesssim 1.0$.

A similar diagram with electrons instead of muons on the external legs also contributes to the electron EDM, $d_{e}$. The experimental constraint $\left|d_{e}\right|<0.105 \times 10^{-26} e \mathrm{~cm}[37]$ translates into $\left|\operatorname{Im}\left(Y_{e \tau} Y_{\tau e}\right)\right|<1.1 \times 10^{-8}$ for a tau running in the loop, and into $\left|\operatorname{Im}\left(Y_{e \mu} Y_{\mu e}\right)\right|<$ $9.8 \times 10^{-8}$ for a muon running in the loop.

\subsection{Constraints from $\mu \rightarrow e$ conversion in nuclei}

Very stringent constraints on the FV Yukawa couplings $Y_{\mu e}$ and $Y_{e \mu}$ come from experimental searches for $\mu \rightarrow e$ conversion in nuclei. The relevant diagrams with one insertion of the FV Yukawa coupling are shown in figure 5. An effective scalar interaction arises already at tree level from the first diagram in figure 5, while vector and electromagnetic dipole contributions arise at one loop level. We give complete expressions for the tree level and one loop contributions in appendix A.3. There are also two-loop contributions, similar to the ones discussed in section 3.1 in the context of $\mu \rightarrow e \gamma$. Numerically, the two-loop contributions are larger than the one loop ones because they are not suppressed by the

\footnotetext{
${ }^{3}$ If the two loop contribution to $\tau \rightarrow \mu \gamma$ is suppressed, e.g. due to a modification of the top Yukawa coupling, which could lead to significant cancellation between the 2-loop top and $W$ diagrams, there is a small region of parameter space in which flavor violating Higgs couplings could explain the $(g-2)_{\mu}$ discrepancy without being ruled out by the one loop $\tau \rightarrow \mu \gamma$ constraint. We will, however, see below that even this case is disfavored by the LHC limit derived in this paper (see section 5.1).
} 


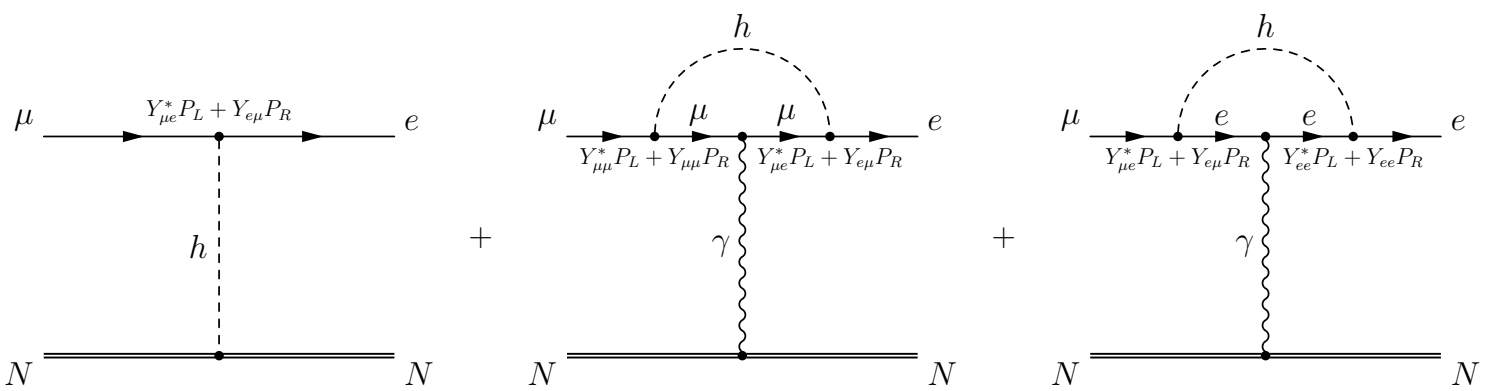

Figure 5. Diagrams contributing to $\mu \rightarrow e$ conversion in nuclei via the flavor violating Higgs Yukawa couplings $Y_{\mu e}$ and $Y_{e \mu}$.

small $Y_{\mu \mu}$ coupling but only by $Y_{t t}$ or the weak gauge coupling, but they are still slightly less than an order of magnitude smaller than the tree level contribution. Here, we always assume the diagonal Yukawa couplings to have their SM values. With this assumption, the tree level term is very sensitive to the strangeness content of the nucleon.

The bounds on the Yukawa couplings $Y_{e \mu}$ and $Y_{\mu e}$ from $\mu \rightarrow e$ conversion in nuclei are listed in table 1.

One could potentially also obtain interesting limits on $\left|Y_{e \tau}\right|$ and $\left|Y_{\tau e}\right|$ from $\mu \rightarrow e$ conversion in nuclei, even though this requires diagrams proportional to two FV Yukawa couplings, because the other constraints on these couplings are weak. The combinations $Y_{e \tau} Y_{\tau \mu}, Y_{e \tau} Y_{\mu \tau}^{*}, Y_{\tau e}^{*} Y_{\tau \mu}$ and $Y_{\tau e}^{*} Y_{\mu \tau}^{*}$ are constrained by $\mu \rightarrow e$ conversion through 1-loop diagrams similar to the ones shown in figure 5, but with a $\tau$ running in the loop (see eq. (A.17)). In the simplest case, $Y_{e \tau}=Y_{\tau e}, Y_{\mu \tau}=Y_{\tau \mu}$, with all Yukawa couplings real, the constraint is $Y_{e \tau} Y_{\mu \tau} \lesssim 10^{-6}$. This is almost, but not quite, competitive with the bound following from $\tau \rightarrow e \gamma$ and $\tau \rightarrow \mu \gamma$ decays, see table 1 .

\subsection{LEP constraints}

The Large Electron-Positron collider (LEP) is indirectly sensitive to the flavor violating Yukawa couplings $Y_{\ell e}$ and $Y_{e \ell}$ (with $\ell=\mu, \tau$ ) through the process $e^{+} e^{-} \rightarrow \ell^{+} \ell^{-}$, mediated by a $t$-channel Higgs. The relevant observables here are the total cross sections $\sigma\left(e^{+} e^{-} \rightarrow \ell^{+} \ell^{-}\right)$and the forward-backward asymmetry of the final state leptons, both of which were measured as a function of the center-of-mass energy $\sqrt{s}$ with uncertainties of order several per cent [45]. However, since the new physics contribution to $\sigma\left(e^{+} e^{-} \rightarrow \ell^{+} \ell^{-}\right)$ is proportional to four powers of the off-diagonal Yukawa couplings, LEP limits cannot compete with constraints from flavor-violating decays like $\tau \rightarrow \mu \gamma$ and $\mu \rightarrow e \gamma$ and with the LHC constraints we derive in section 5. While a full derivation of LEP limits, including a careful treatment of the interference between Standard Model and non-standard contributions as well as a fit to the data points given in [45] is beyond the scope of this work, we have estimated that flavor-violating couplings $\sqrt{\left|Y_{\ell e}\right|^{2}+\left|Y_{e \ell}\right|^{2}} \lesssim$ few $\times 10^{-1}$ are excluded by LEP. 


\subsection{Allowed branching ratios for lepton flavor violating Higgs decays}

In figure 6 we collect the above constraints on the values of $\left|Y_{e \tau}\right|,\left|Y_{\tau e}\right|$ (upper left panel), $\left|Y_{e \mu}\right|,\left|Y_{\mu e}\right|$ (upper right panel) and $\left|Y_{\mu \tau}\right|,\left|Y_{\tau \mu}\right|$ (lower panel) and relate them to the predicted branching ratios for $h \rightarrow e \tau, h \rightarrow e \tau$ and $h \rightarrow \mu \tau$. The latter are given by

$$
\operatorname{BR}\left(h \rightarrow \ell^{\alpha} \ell^{\beta}\right)=\frac{\Gamma\left(h \rightarrow \ell^{\alpha} \ell^{\beta}\right)}{\Gamma\left(h \rightarrow \ell^{\alpha} \ell^{\beta}\right)+\Gamma_{\mathrm{SM}}},
$$

where $\ell^{\alpha}, \ell^{\beta}=e, \mu, \tau, \ell^{\alpha} \neq \ell^{\beta}$. The decay width $\Gamma\left(h \rightarrow \ell^{\alpha} \ell^{\beta}\right)$, in turn, is

$$
\Gamma\left(h \rightarrow \ell^{\alpha} \ell^{\beta}\right)=\frac{m_{h}}{8 \pi}\left(\left|Y_{\ell^{\beta} \ell^{\alpha}}\right|^{2}+\left|Y_{\ell^{\alpha} \ell^{\beta}}\right|^{2}\right),
$$

and the SM Higgs width is $\Gamma_{\mathrm{SM}}=4.1 \mathrm{MeV}$ for a $125 \mathrm{GeV}$ Higgs boson [46]. In the panels of figure 6 we are assuming that at most one of non-standard decay mode of the Higgs is significant compared to the SM decay width.

From figure 6 we see that given current bounds from $\tau \rightarrow \mu \gamma$ and $\tau \rightarrow e \gamma$, branching fractions for $h \rightarrow \tau \mu$ or $h \rightarrow \tau e$ in the neighborhood of $10 \%$ are allowed. This is well within the reach of the LHC as we shall show in section 5. The allowed sizes of these two decay widths are comparable to the sizes of decay widths into nonstandard decay channels (such as the invisible decay width) that are allowed by global fits [47]. If there is no significant negative contribution to Higgs production through gluon fusion, one has $\mathrm{BR}(h \rightarrow$ invisible $) \lesssim 20 \%$, while allowing for arbitrarily large modifications of gluon and photon couplings to the Higgs leads to the constraint $\mathrm{BR}(h \rightarrow$ invisible $) \lesssim 65 \%$ [47]. These two bounds apply without change also to $\mathrm{BR}(h \rightarrow \tau \mu), \mathrm{BR}(h \rightarrow \tau e)$ and $\mathrm{BR}(h \rightarrow e \mu)$.

In contrast to decays involving a $\tau$ lepton, the branching ratio for $h \rightarrow e \mu$ is extremely well constrained by $\mu \rightarrow e \gamma, \mu \rightarrow 3 e$ and $\mu \rightarrow e$ conversion bounds, and is required to be below $\mathrm{BR}(h \rightarrow e \mu) \lesssim 2 \times 10^{-8}$, well beyond the reach of the LHC.

\section{Hadronic flavor violating decays of the Higgs}

We next consider flavor violating decays of the Higgs to quarks. We first discuss two-body decays to light quarks, $h \rightarrow \bar{b} d, \bar{b} s, \bar{s} d, \bar{c} u$, and then turn to FV three body decays mediated by an off-shell top, $h \rightarrow \bar{t}^{*} c \rightarrow W \bar{b} c$ and $h \rightarrow \bar{t}^{*} u \rightarrow W \bar{b} u$ as well as FV top decays to $t \rightarrow c h$ and $t \rightarrow u h$. Our limits are summarized in table 2 .

\subsection{Flavor violating Higgs decays into light quarks}

Flavor violating Higgs couplings to quarks can generate flavor changing neutral currents (FCNCs) at tree level, see figure 7 (a), and are thus well constrained by the measured $B_{d, s}-\bar{B}_{d, s}, K^{0}-\bar{K}^{0}$ and $D^{0}-\bar{D}^{0}$ mixing rates. Integrating out the Higgs generates an effective weak Hamiltonian, which for $B_{d}-\bar{B}_{d}$ mixing is

$$
H_{\mathrm{eff}}=C_{2}^{d b}\left(\bar{b}_{R} d_{L}\right)^{2}+\tilde{C}_{2}^{d b}\left(\bar{b}_{L} d_{R}\right)^{2}+C_{4}^{d b}\left(\bar{b}_{L} d_{R}\right)\left(\bar{b}_{R} d_{L}\right) .
$$



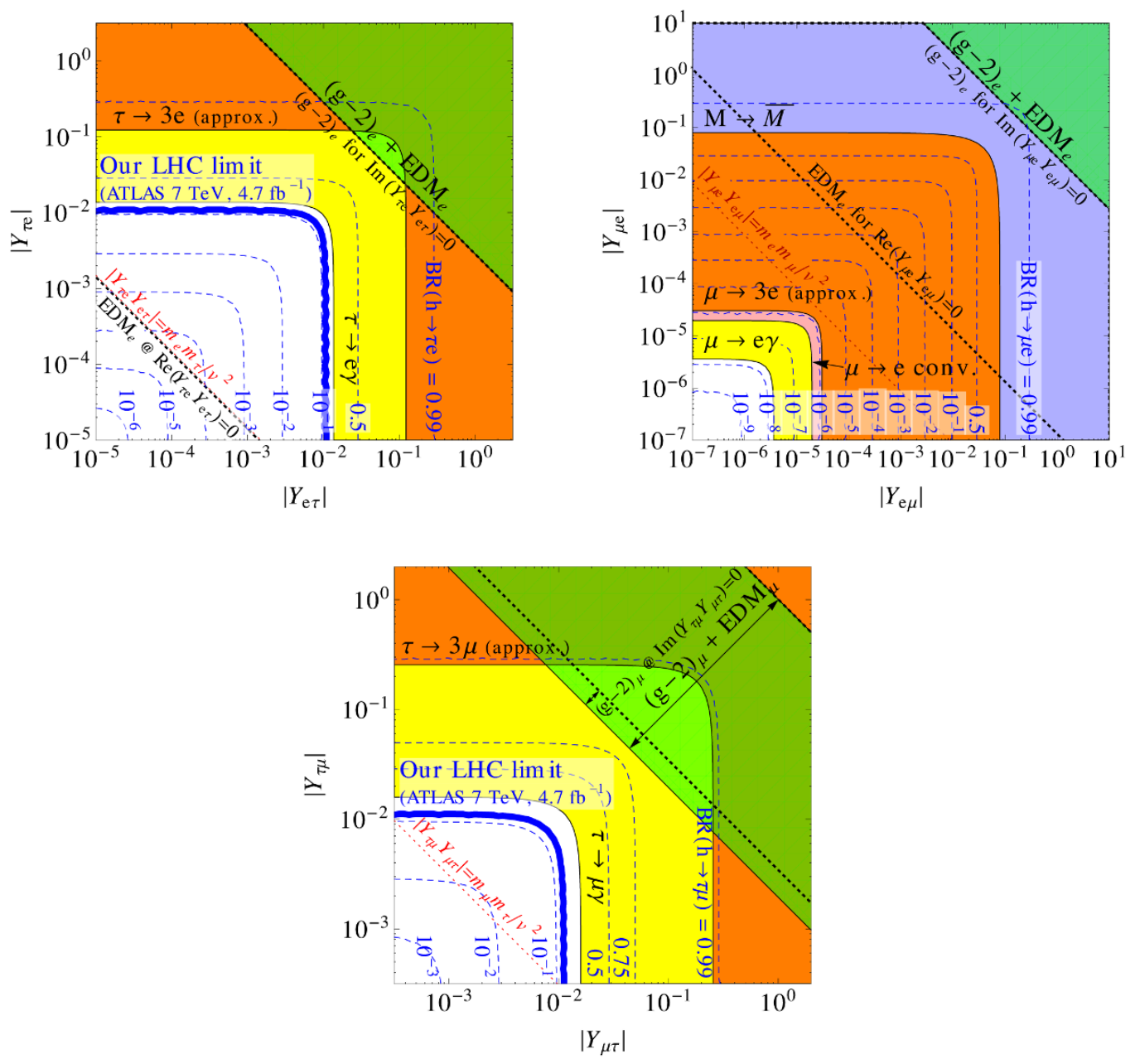

Figure 6. Constraints on the flavor violating Yukawa couplings $\left|Y_{e \tau}\right|,\left|Y_{\tau e}\right|$ (upper left panel), $\left|Y_{e \mu}\right|$, $\left|Y_{\mu e}\right|$ (upper right panel) and $\left|Y_{\mu \tau}\right|,\left|Y_{\tau \mu}\right|$ (lower panel) of a $125 \mathrm{GeV}$ Higgs boson. The diagonal Yukawa couplings are approximated by their SM values. Thin blue dashed lines are contours of constant BR for $h \rightarrow \tau e, h \rightarrow \mu e$ and $h \rightarrow \tau \mu$, respectively, whereas thick blue lines are the LHC limits derived in section 5.1. (These limits could be greatly improved with dedicated searches on existing LHC data, see section 5.3.) Shaded regions show the constraints discussed in section 3 as indicated in the plots. Note that $g-2[\mathrm{EDM}]$ searches (diagonal black dotted lines) are only sensitive to parameter combinations of the form $\operatorname{Re}\left(Y_{\alpha \beta} Y_{\beta \alpha}\right)\left[\operatorname{Im}\left(Y_{\alpha \beta} Y_{\beta \alpha}\right)\right]$. We also show limits from a combination of $g-2$ and EDM searches with marginalization over the complex phases of the Yukawa couplings (green shaded regions). Note that $(g-2)_{\mu}$ provides upper and lower limits (as indicated by the double-sided arrows in the lower panel) if the discrepancy between the measurement and the SM prediction $[38,43]$ is taken into account. The thin red dotted lines show rough naturalness limits $Y_{i j} Y_{j i} \lesssim m_{i} m_{j} / v^{2}$ (see section 2).

Here we use the same notation for the Wilson coefficients as in [48] and display only nonzero contributions, which are

$$
C_{2}^{d b}=-\frac{\left(Y_{d b}^{*}\right)^{2}}{2 m_{h}^{2}}, \quad \tilde{C}_{2}^{d b}=-\frac{\left(Y_{b d}^{2}\right)^{2}}{2 m_{h}^{2}}, \quad C_{4}^{d b}=-\frac{Y_{b d} Y_{d b}^{*}}{m_{h}^{2}} .
$$




\begin{tabular}{|ccc|}
\hline Technique & Coupling & Constraint \\
\hline$D^{0}$ oscillations [48] & $\left|Y_{u c}\right|^{2},\left|Y_{c u}\right|^{2}$ & $<5.0 \times 10^{-9}$ \\
& $\left|Y_{u c} Y_{c u}\right|$ & $<7.5 \times 10^{-10}$ \\
\hline \multirow{2}{*}{$B_{d}^{0}$ oscillations [48] } & $\left|Y_{d b}\right|^{2},\left|Y_{b d}\right|^{2}$ & $<2.3 \times 10^{-8}$ \\
& $\left|Y_{d b} Y_{b d}\right|$ & $<3.3 \times 10^{-9}$ \\
\hline \multirow{2}{*}{$B_{s}^{0}$ oscillations [48] } & $\left|Y_{s b}\right|^{2},\left|Y_{b s}\right|^{2}$ & $<1.8 \times 10^{-6}$ \\
& $\left|Y_{s b} Y_{b s}\right|$ & $<2.5 \times 10^{-7}$ \\
\hline \multirow{2}{*}{$K^{0}$ oscillations [48] } & $\operatorname{Re}\left(Y_{d s}^{2}\right), \operatorname{Re}\left(Y_{s d}^{2}\right)$ & {$[-5.9 \ldots 5.6] \times 10^{-10}$} \\
& $\operatorname{Im}\left(Y_{d s}^{2}\right), \operatorname{Im}\left(Y_{s d}^{2}\right)$ & {$[-2.9 \ldots 1.6] \times 10^{-12}$} \\
& $\operatorname{Re}\left(Y_{d s}^{*} Y_{s d}\right)$ & {$[-5.6 \ldots 5.6] \times 10^{-11}$} \\
single-top production [49] & $\operatorname{Im}\left(Y_{d s}^{*} Y_{s d}\right)$ & {$[-1.4 \ldots 2.8] \times 10^{-13}$} \\
& $\sqrt{\left|Y_{t c}^{2}\right|+\left|Y_{c t}\right|^{2}}$ & $<3.7$ \\
\hline \multirow{2}{*}{$t \rightarrow h j[50]$} & $\sqrt{\left|Y_{t u}^{2}\right|+\left|Y_{u t}\right|^{2}}$ & $<1.6$ \\
\hline & $\sqrt{\left|Y_{t c}^{2}\right|+\left|Y_{c t}\right|^{2}}$ & $<0.34$ \\
& $\sqrt{\left|Y_{t u}^{2}\right|+\left|Y_{u t}\right|^{2}}$ & $<0.34$ \\
\hline$D^{0}$ oscillations [48] & $\left|Y_{u t} Y_{c t}\right|,\left|Y_{t u} Y_{t c}\right|$ & $<7.6 \times 10^{-3}$ \\
& $\left|Y_{t u} Y_{c t}\right|,\left|Y_{u t} Y_{t c}\right|$ & $<2.2 \times 10^{-3}$ \\
\hline neutron EDM [37] & $\left|Y_{u t} Y_{t u} Y_{c t} Y_{t c}\right|^{1 / 2}$ & $<0.9 \times 10^{-3}$ \\
\hline & $\operatorname{Im}\left(Y_{u t} Y_{t u}\right)$ & $<4.4 \times 10^{-8}$ \\
\hline
\end{tabular}

Table 2. Constraints on flavor violating Higgs couplings to quarks. We have assumed a Higgs mass $m_{h}=125 \mathrm{GeV}$, and we have taken the diagonal Yukawa couplings at their SM values.

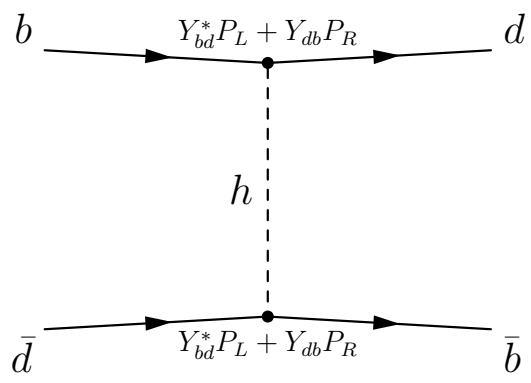

(a)

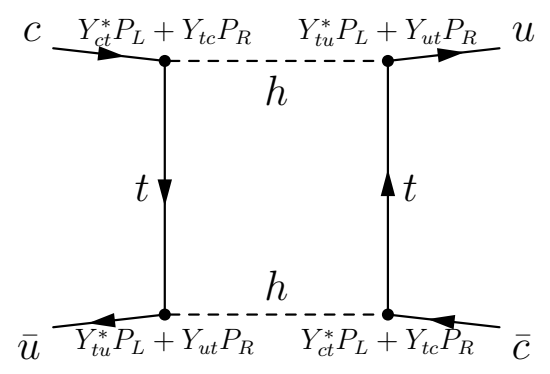

(b)

Figure 7. Two representative diagrams through which flavor violating Higgs Yukawa couplings can contribute to neutral meson mixing.

The results for $B_{s}-\bar{B}_{s}, K^{0}-\bar{K}^{0}$ and $D^{0}-\bar{D}^{0}$ mixing are obtained in the same way with the obvious quark flavor replacements. We can now translate the bounds on the above Wilson coefficients obtained in [48] into constraints on the combinations of flavor violating 
Higgs couplings as summarized in table 2. We see that all Yukawa couplings involving only $u, d, s, c$, or $b$ quarks have to be tiny. The weakest constraints are those in the $b-s$ sector, where flavor violating Yukawa couplings $\lesssim 10^{-3}$ are still allowed. This would correspond to $\mathrm{BR}(h \rightarrow b s) \sim 2 \times 10^{-3}$, which is still far too small to be observed at the LHC because of the large QCD backgrounds.

\subsection{Higgs decays through off-shell top and top decays to Higgs}

Among the flavor violating Higgs couplings to quarks, the most promising place for new physics to hide are processes involving top quarks, such as the 3-body decay $h \rightarrow\left(t^{*} \rightarrow\right.$ $W b) q$. Here, $q$ denotes either a charm quark or an up quark. The corresponding FV Yukawa couplings contribute at one loop to $D-\bar{D}$ mixing through diagrams of the form of figure 7 (b). The corresponding Wilson coefficients in the effective Hamiltonian (4.1) are

$$
\begin{aligned}
C_{1}^{u c} & =\frac{1}{4} \frac{1}{16 \pi^{2}} \frac{S_{1}^{H}\left(x_{t H}\right)}{m_{h}^{2}}\left(Y_{c t} Y_{u t}^{*}\right)^{2}, & \tilde{C}_{1}^{u c} & =\frac{1}{4} \frac{1}{16 \pi^{2}} \frac{S_{1}^{H}\left(x_{t H}\right)}{m_{h}^{2}}\left(Y_{t c}^{*} Y_{t u}\right)^{2}, \\
C_{2}^{u c} & =-\frac{1}{4} \frac{1}{16 \pi^{2}} \frac{S_{2}^{H}\left(x_{t H}\right)}{m_{h}^{2}}\left(Y_{t c}^{*} Y_{u t}^{*}\right)^{2}, & \tilde{C}_{2}^{u c} & =-\frac{1}{4} \frac{1}{16 \pi^{2}} \frac{S_{2}^{H}\left(x_{t H}\right)}{m_{h}^{2}}\left(Y_{c t} Y_{t u}\right)^{2}, \\
C_{4}^{u c} & =-\frac{1}{2} \frac{1}{16 \pi^{2}} \frac{S_{2}^{H}\left(x_{t H}\right)}{m_{h}^{2}}\left(Y_{c t} Y_{t u}\right)\left(Y_{t c}^{*} Y_{u t}^{*}\right), & C_{5}^{u c} & =-\frac{1}{16 \pi^{2}} \frac{S_{1}^{H}\left(x_{t H}\right)}{m_{h}^{2}}\left(Y_{c t} Y_{u t}^{*}\right)\left(Y_{t c}^{*} Y_{t u}\right),
\end{aligned}
$$

where

$$
S_{1}^{H}(x)=\frac{x^{2}-1-2 x \log x}{2(x-1)^{3}}, \quad S_{2}^{H}(x)=\frac{2 x[2-2 x+(1+x) \log x]}{(x-1)^{3}},
$$

and $x_{t H} \equiv m_{t}^{2} / m_{h}^{2}$. Note that now also the operators $Q_{1,5}^{u c}, \tilde{Q}_{1}^{u c}$ (in the notation of [48]) have non-zero Wilson coefficients. By requiring that each individual operator is consistent with its $D-\bar{D}$ mixing constraint, we derive the limits shown in the last part of table 2 . The constraints are much weaker than those on FV Higgs couplings involving only light quarks.

Strong constraints on $Y_{q t}$ and $Y_{t q}$ are also obtained from the non-observation of anomalous single top production. The flavor violating chromomagnetic operators

$$
\mathcal{L}_{\text {single top }} \supset \frac{g_{s}}{m_{h}} \bar{t} \sigma^{\mu \nu}\left(\kappa_{t q g, L} P_{L}+\kappa_{t q g, R} P_{R}\right) \frac{\lambda^{a}}{2} q G_{\mu \nu}^{a},
$$

are generated trough loop diagrams similar to figure 1, but with leptons replaced by quarks and the photon replaced by a gluon. Here $g_{s}$ is the strong coupling constant, $\lambda^{a}$ are the GellMann matrices, $G_{\mu \nu}^{a}$ is the gluon field strength tensor, and $\kappa_{t q g, L}, \kappa_{t q g, R}$ are dimensionless effective coupling constants which depend on $Y_{q t}$ and $Y_{t q}$ according to

$$
\kappa_{t q g, L}=\frac{m_{t} m_{h}}{8 \pi^{2}} F\left(m_{t}, m_{t}, 0, q^{2}=0, Y^{\dagger}\right)
$$

with the loop function $F$ given in eq. (A.3). The analogous expression for $\kappa_{t q g, R}$ is obtained by replacing $Y_{t q}^{*} \rightarrow Y_{q t}$ and $Y_{t t} \rightarrow Y_{t t}^{*}$ in $F$. Note that in (4.8) we have assumed an EFT 


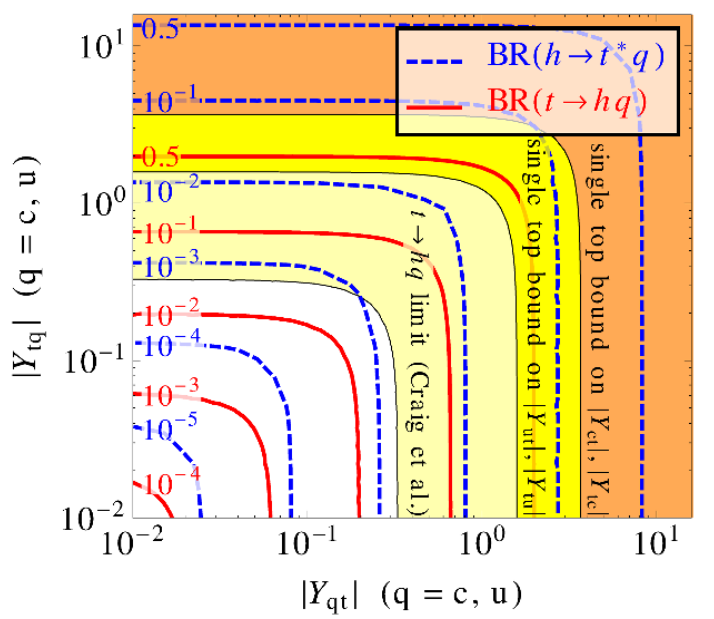

Figure 8. Predictions for various flavor changing neutral current (FCNC) processes mediated by the flavor violating Yukawa couplings $Y_{c t}, Y_{t c}$ or $Y_{u t}, Y_{t u}$ of a $125 \mathrm{GeV}$ Higgs boson. Where appropriate, we have approximated the diagonal Yukawa couplings by their Standard Models values. Blue dashed contours indicate the branching ratio for $h \rightarrow t^{*} q$, red solid contours the one for $t \rightarrow h q$ (where $q$ denotes a charm or up quark). The light yellow region shows a recent limit on $t \rightarrow h c$ (or $h u$ ) from an LHC multi-lepton search [50].

description with an on-shell gluon. Since $m_{h} \sim m_{t}$ this is only approximate, but we have checked that varying $q^{2} \in\left[0, m_{t}^{2}\right]$ changes the bounds on $Y_{t q}, Y_{q t}$ only by $\sim 10 \%$. We have also made the approximation $m_{q} \rightarrow 0$, which is obeyed even much better. Limits on $\kappa_{t q g, L}$, $\kappa_{t q g, R}$ have been derived by the CDF and D $\varnothing$ collaborations $[49,51]$ and most recently by ATLAS [52]. In the notation of [52], we have $\left|\kappa_{t g f}\right| / \Lambda \equiv \sqrt{\left|\kappa_{t q g, L}\right|^{2}+\left|\kappa_{t q g, R}\right|^{2}} /\left(\sqrt{2} m_{h}\right)$. We obtain the constraints

$$
\sqrt{\left|Y_{t c}^{2}\right|+\left|Y_{c t}\right|^{2}}<3.7, \quad \sqrt{\left|Y_{t u}^{2}\right|+\left|Y_{u t}\right|^{2}}<1.6,
$$

We now translate these bounds into constraints on the $h \rightarrow\left(\bar{t}^{*} \rightarrow W \bar{b}\right) q$ decay width, which is given by (setting $m_{b, q}=0$ )

$$
\begin{aligned}
\frac{d^{2} \Gamma\left(h \rightarrow \bar{t}^{*} q\right)}{d m_{12}^{2} d m_{23}^{2}}= & \frac{3 g^{2}\left|V_{t b}\right|^{2}}{64(2 \pi)^{3} m_{W}^{2} m_{h}^{3}} \frac{1}{\left(m_{23}^{2}-m_{t}^{2}\right)^{2}}\left[m_{12}^{2}\left(2 m_{W}^{2}-m_{23}^{2}\right)\left(m_{t}^{2}\left|Y_{q t}\right|^{2}-m_{23}^{2}\left|Y_{t q}\right|^{2}\right)\right. \\
& \left.+\left(m_{h}^{2}-m_{23}^{2}\right)\left(m_{23}^{2}-m_{W}^{2}\right)\left(2 m_{W}^{2}\left|Y_{t q}\right|^{2}+m_{t}^{2}\left|Y_{q t}\right|^{2}\right)\right]
\end{aligned}
$$

where $V_{t b} \simeq 1$ is a CKM matrix element. The branching ratio for $h \rightarrow t^{*} c$ can be as large as $\mathcal{O}\left(10^{-3}\right)$, and the one for $h \rightarrow t^{*} u$ can be few $\times 10^{-4}$ as shown in figure 8 .

If the decay $h \rightarrow\left(t^{*} \rightarrow W b\right) c$ is non-negligible, so is the related non-standard top quark decay mode $t \rightarrow h c$, the rate for which is given by (neglecting the charm mass)

$$
\Gamma(t \rightarrow h c)=\frac{\left|Y_{c t}\right|^{2}+\left|Y_{t c}\right|^{2}}{32 \pi} \frac{\left(m_{t}^{2}-m_{h}^{2}\right)^{2}}{m_{t}^{3}} .
$$

Branching ratios for $t \rightarrow h c$ of several tens of per cent are perfectly viable and can be searched for, e.g. in the multi-lepton or $t \rightarrow b \bar{b} c$ channels. In fact, the strongest limit on 
Higgs couplings to tc are already coming from a CMS multi-lepton search which was recast in [50] to search for $t \rightarrow h c$, giving a bound of $2.7 \%$ on the branching fraction of a top into a Higgs and a charm or up quark. This yields a limit of $\sqrt{\left|Y_{t i}\right|^{2}+\left|Y_{i t}\right|^{2}}<0.34$ for $i=u$ or $c$ (see figure 8).

We have also calculated the branching ratios for the loop-induced processes $t \rightarrow q \gamma$, $t \rightarrow q g$ and $t \rightarrow q Z(q=u, c)$, which are in principle sensitive to $\left|Y_{q t}\right|$ and $\left|Y_{t q}\right|$, but have found that even for $\left|Y_{q t}\right|,\left|Y_{t q}\right| \sim \mathcal{O}(1)$ the current experimental bounds are satisfied [53].

In the above we have assumed that the weak phases of $Y_{u t}$ and $Y_{t u}$ are negligibly small. Otherwise an unacceptably large contribution to the neutron EDM is generated at 1-loop level with top and Higgs running in the loop. Eq. (3.17) with the replacements $m_{\tau} \rightarrow m_{t}$, and $Y_{\mu \tau} Y_{\tau \mu} \rightarrow Y_{u t} Y_{t u}$ gives the $u$-quark EDM $d_{u}=e \tilde{d}_{u}$, from which one can calculate the neutron EDM $d_{n}$ [54]. Using the 90\% CL experimental bound $d_{n}<$ $0.29 \times 10^{-25} \mathrm{ecm}$ [37] together with the theoretical prediction, eq. (3.62) of [54], one obtains $\left|\operatorname{Im} Y_{u t} Y_{t u}\right|<4.4 \times 10^{-8}$, which is much more stringent than the bounds on the absolute values of the same FV Yukawa couplings. In contrast, the bounds from charm running in the loop, $\left|\operatorname{Im} Y_{u c} Y_{c u}\right|<1.6 \times 10^{-7}$, and from $d$-quark EDMs generated by the $b$-quark and $s$-quark running in the loop, $\left|\operatorname{Im} Y_{d b} Y_{b d}\right|<6.4 \times 10^{-8}$ and $\left|\operatorname{Im} Y_{d s} Y_{s d}\right|<1.2 \times 10^{-6}$, respectively, are less stringent than the bounds from meson mixing, table 2.

\section{Searching for flavor violating Higgs decays at the LHC}

We next discuss possible search strategies for flavor violating Higgs decays at the LHC, focusing on the $h \rightarrow \tau \mu$ and $h \rightarrow \tau e$ decays. As shown in figure 6, these are among the least constrained of the couplings discussed in this paper, with a potential to modify the Higgs branching fractions significantly. They are sensitive to new particles with flavor violating couplings or to a secondary mechanisms of electroweak symmetry breaking such as additional Higgs doublets, and are thus good probes of new physics. Furthermore, they are also interesting final states as far as the potential for searches at the LHC is concerned.

The decay $h \rightarrow \tau \mu$ is quite similar to the standard model $h \rightarrow \tau \tau$ decay with one of the tau leptons decaying to a muon. This implies that existing SM Higgs searches, with only small or no modifications at all, can already be used to place bounds on the flavor violating decay. We thus first extract limits on $h \rightarrow \tau \mu$ and $h \rightarrow \tau e$ decays from an existing $h \rightarrow \tau \tau$ search in ATLAS. We then discuss how modifications to the $\tau \tau$ search can lead to significantly improved sensitivity to flavor violating Higgs decays.

\subsection{Extracting a bound on Higgs decays to $\tau \mu$ and $\tau e$}

We use the existing ATLAS search for $h \rightarrow \tau \tau$ in the fully leptonic channel [55] to place bounds on the $h \rightarrow \tau \mu$ and $h \rightarrow \tau e$ branching fractions. The reason we use fully leptonic events is that we can simulate the detector response to them more accurately than for events involving hadronic taus. It should, however, be noted that in the SM $h \rightarrow \tau \tau$ search in ATLAS, semi-hadronic events are about as sensitive as fully leptonic ones [55], and in CMS, the semi-hadronic mode provides even stronger limits [27]. The analysis in [55] uses the collinear approximation to reconstruct the $\tau \tau$ invariant mass, i.e. it is assumed that the 
neutrino and the charged lepton emitted in tau decay are collinear. This approach is less optimized for $h \rightarrow \tau \tau$ than the maximum-likelihood method employed by CMS [56], but it is more model independent so that a substantial fraction of $h \rightarrow \tau \mu$ or $h \rightarrow \tau e$ decays would pass the cuts. ${ }^{4}$ For simplicity we only use the ATLAS cuts optimized for Higgs production in vector boson fusion (VBF) since this channel provides the best sensitivity [55].

To derive limits we have generated 50,000 $p p \rightarrow 2 j+(h \rightarrow \tau \mu)$ Monte Carlo events using MadGraph 5 v1.4.6 [57] for parton level event generation, Pythia 6.4 for parton showering and hadronization, and PGS [58] as a fast detector simulation. Combining the ATLAS lepton triggers and off-line cuts from [55], we select opposite sign dilepton events satisfying any of the following requirements: a muon pair with $p_{T}>15 \mathrm{GeV}$ for the leading muon and $p_{T}>10 \mathrm{GeV}$ for the subleading one, an electron pair with both $p_{T}>15 \mathrm{GeV}$, or an electron and a muon with $p_{T}$ above 15 and $10 \mathrm{GeV}$, respectively. Electrons (muons) are accepted only if their pseudorapidity is $|\eta|<2.47(2.5)$. We require the invariant mass of the lepton pair to be $30 \mathrm{GeV}<m_{l \bar{l}}<100 \mathrm{GeV}$ for $e \mu$ pairs, or $30 \mathrm{GeV}<m_{l \bar{l}}<75 \mathrm{GeV}$ for same flavor pairs. The missing $p_{T}$ is required to be above 20 (40) GeV for $e \mu$ events (ee or $\mu \mu$ events). The azimuthal separation between the two leptons is required to be $0.5<\Delta \phi_{l l}<2.5$.

Additional cuts are placed with the goal of enriching the event sample in VBF events: at least two jets with $p_{T}$ above $40 \mathrm{GeV}$ for the leading jet and above $25 \mathrm{GeV}$ for the subleading jet are required, with the rapidity difference between the two leading jets above $|\Delta \eta|>3$ and the invariant mass $m_{j j}>350 \mathrm{GeV}$. We veto events with an additional jet with $p_{T}>25 \mathrm{GeV}$ and $|\eta|<2.4$ in the pseudorapidity region between the two leading jets.

The reconstructed invariant mass is calculated using the collinear approximation in which all invisible particles are assumed to be collinear with either of the two leptons. The fractions of the parent $\tau$ 's momenta carried by the charged leptons are denoted by $x_{1}$ and $x_{2}$. To be able to compare with ATLAS data from the fully leptonic $h \rightarrow \tau \tau$ search, we compute $x_{1}$ and $x_{2}$ assuming four neutrinos in the final state, even though fully leptonic $h \rightarrow \tau \mu$ decays yield only two. $x_{1}$ and $x_{2}$ are then obtained as the solutions of the transverse momentum equation $\mathbf{p}_{\text {miss }, T}=\left(1-x_{1}\right) \mathbf{p}_{1, T}+\left(1-x_{2}\right) \mathbf{p}_{2, T}$, where $\mathbf{p}_{1,2, T}$ are the transverse momenta of the charged leptons. Following [55], we require $0.1<x_{1,2}<1$, which removes less than a per cent of $h \rightarrow \tau \tau$ events, but nearly $60 \%$ of our $h \rightarrow \tau \mu$ events. Thus, relaxing this cut would enhance the sensitivity to $h \rightarrow \tau \mu$ decays so long as it does not introduce large backgrounds. Nonetheless, we are still able to use the current search for $h \rightarrow \tau \tau$ to produce an interesting bound on $\operatorname{BR}(h \rightarrow \tau \mu)$.

In figure 9 we show the background distribution for the collinear mass along with the expected shape of a LFV $h \rightarrow \tau \mu$ signals (scaled by a factor five for illustrative purposes only), and we compare to the observed data. The background expectation is taken from [55]. The backgrounds and the data in figure 9 include events for all three combinations of lepton flavor (even though our $\tau \mu$ signal does not induce ee events) because only this information is available from ATLAS. For validation purposes, we have also simulated

\footnotetext{
${ }^{4}$ In fact, it may be interesting to apply the collinear approximation more often in resonance searches. A search for a collinear mass resonance can be sensitive to any particle which decays to boosted objects whose further decay may introduce missing energy.
} 


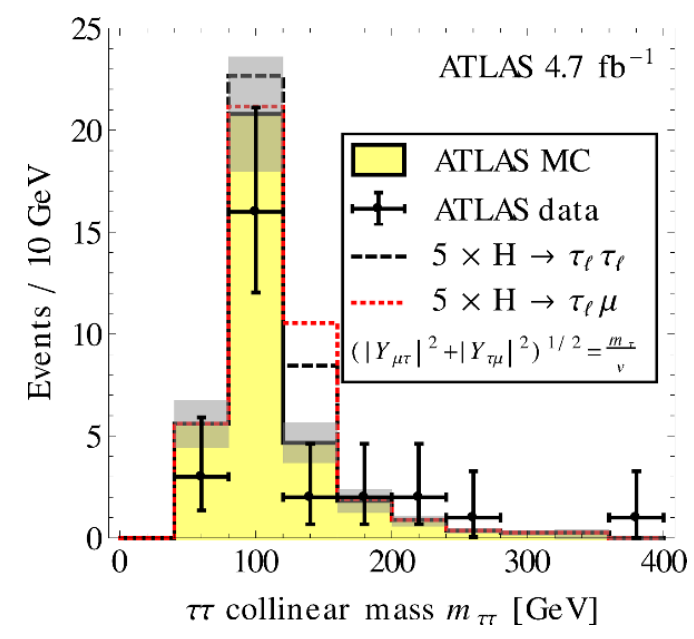

Figure 9. Background rates and $h \rightarrow \tau \mu, h \rightarrow \tau \tau$ signal rates in the ATLAS search for fully leptonic $h \rightarrow \tau \tau$ decays, optimized for Higgs production in vector boson fusion. The backgrounds expected by ATLAS [55] are shown in yellow, with grey bands for the systematic uncertainty. Our estimates for the $\tau \mu$ signal at $\sqrt{Y_{\tau \mu}^{2}+Y_{\mu \tau}^{2}}=m_{\tau} / v$ (red) and the SM $h \rightarrow \tau \tau$ signal (black), which we include for reference, are scaled by a factor 5 for illustrative purposes only.

\begin{tabular}{|ccccc|}
\hline 95\% C.L. limit & $\mathrm{BR}(h \rightarrow \tau \mu)$ & $\sqrt{Y_{\tau \mu}^{2}+Y_{\mu \tau}^{2}}$ & $\mathrm{BR}(h \rightarrow \tau e)$ & $\sqrt{Y_{\tau e}^{2}+Y_{e \tau}^{2}}$ \\
\hline expected & $28 \%$ & 0.018 & $27 \%$ & 0.017 \\
observed & $13 \%$ & 0.011 & $13 \%$ & 0.011 \\
\hline
\end{tabular}

Table 3. Expected and observed 95\% C.L. limits on the $h \rightarrow \tau \mu$ and $h \rightarrow \tau e$ branching ratios, as well as limits on the corresponding Yukawa couplings. The limits are derived by assuming the SM Higgs production rates and recasting the search for SM $h \rightarrow \tau \tau \rightarrow 2 \ell+2 \nu$ decays in the VBF channel from [55].

SM $h \rightarrow \tau \tau$ events, and comparing the rate and shape to ref. [55] we find agreement to within $20 \%$.

The $\tau \mu$ signal is predominantly concentrated in the $120-160 \mathrm{GeV}$ bin, so that the expected and observed limits on the flavor violating Yukawa couplings can be derived from a simple single-bin analysis. If we denote the number of expected background events by $B=4.7$, the number of expected signal events for a given set of Yukawa couplings by $S$, and the number of observed events by $O=2$, the expected (observed) one-sided 95\% C.L. frequentist limit on $S$ is defined by the requirement that the probability to observe $\leq B$ $(\leq O)$ events is $5 \%$. The relevant probability distribution of the data here is a Poisson distribution with mean $B+S$. We can also include the systematic uncertainty in the $120-160 \mathrm{GeV}$ bin, which is $\Delta_{\text {sys }} \simeq \pm 0.99$, in a conservative way by instead using a Poisson distribution with mean $B+S-\Delta_{\text {sys }}$. Assuming the Higgs is produced with the Standard Model rates, this procedure leads to the bound on $\operatorname{BR}(h \rightarrow \tau \mu)$ and the analogous bound on $\mathrm{BR}(h \rightarrow \tau e)$ shown in table 3 (see also figure 6). 


\subsection{Comparison of $h \rightarrow \tau \mu$ to $h \rightarrow \tau \tau$}

We now discuss the experimental differences and similarities between $h \rightarrow \tau \tau$ and $h \rightarrow \tau \mu$ decays to determine an optimized search strategy for the latter. We focus here on $h \rightarrow$ $\tau_{\text {had }} \tau_{\mu}$,where $\tau_{\mu}$ denotes a $\tau$ that decays into a muon and two neutrinos and $\tau_{\text {had }}$ denotes a $\tau$ decaying hadronically. This channel is actively searched for, both at ATLAS [55] and at CMS [27], and is the most sensitive channel in the CMS $h \rightarrow \tau \tau$ search. (In ATLAS, fully leptonic $\tau$ events provide similar sensitivity to semi-hadronic ones.) It will also be the channel that we will devise a dedicated search for in the next subsection.

There are a few notable differences between the $h \rightarrow \tau_{\text {had }} \tau_{\mu}$ and $h \rightarrow \tau_{\text {had }} \mu$ decay channels:

- Branching Ratios. The branching fraction for $h \rightarrow \tau_{\text {had }} \tau_{\mu}$ is $2 \times \mathrm{BR}(h \rightarrow \tau \tau) \times \mathrm{BR}(\tau \rightarrow$ had $) \times \operatorname{BR}(\tau \rightarrow \mu)$, whereas for $h \rightarrow \tau_{\text {had }} \mu$ it is simply $\operatorname{BR}(h \rightarrow \tau \mu) \times \operatorname{BR}(\tau \rightarrow$ had $)$. For $\left(Y_{\tau \mu}^{2}+Y_{\mu \tau}^{2}\right)^{1 / 2} \sim Y_{\tau \tau}$ the signal for $h \rightarrow \tau_{\text {had }} \mu$ is thus a factor of $\sim 2.9$ larger.

- Lepton Flavor. The flavor violating decays can lead to different rates for muons and electrons in the final state, whereas $\tau \tau$ decays lead to equal $\mu$ and $e$ rates. Thus, if the various lepton flavor combinations were studied separately in the $h \rightarrow \tau \tau$ analyses, stronger bounds on flavor violating decays could be inferred.

- Kinematics and Efficiencies. In $h \rightarrow \tau_{\text {had }} \tau_{\mu}$ decays the muon carries an average energy $\sim m_{h} / 6$, while for $h \rightarrow \tau_{\text {had }} \mu$ it carries $\sim m_{h} / 2$. Furthermore, in $h \rightarrow \tau_{\text {had }} \mu$ events the missing energy is roughly aligned with the hadronic $\tau$. As a result the two channels can have different efficiencies given the same cuts. For example, in the VBF analysis described below (mimicking [27]) the efficiency for $h \rightarrow \tau_{\text {had }} \tau_{\mu}$ is a factor of $\sim 1.8$ lower than for $h \rightarrow \tau_{\text {had }} \mu$ events, mostly because many of the muons in the $h \rightarrow \tau_{\text {had }} \tau_{\mu}$ sample fall below the $p_{T}<17 \mathrm{GeV}$ cut.

- Mass reconstruction. The LHC collaborations use highly optimized procedures for reconstructing the $\tau_{\text {had }} \tau_{\mu}$ invariant mass. ATLAS uses the Missing Mass Calculator (MMC) from [56], while CMS uses an in-house maximum likelihood analysis [27]. These procedures use $\mathbf{p}_{\text {miss, } T}$ and the 3 -momenta of the muon and the $\tau$ jet as input and estimate the neutrino momenta by assuming typical $\tau$ decay kinematics. For $h \rightarrow \tau \tau$ events, the MMC procedure returns an invariant mass with high efficiency $(\sim 97 \%)$ and gives a Higgs mass resolution of $\sim 20 \%$. If the event is not from $h \rightarrow \tau \tau$ but instead from $h \rightarrow \tau_{\text {had }} \mu$, then i) the efficiency will be significantly lower since the kinematics can be completely inconsistent with a $\tau \tau$ event, and ii) the reconstructed Higgs mass will be significantly higher as the MMC will assume that the hard muon is accompanied by two roughly collinear and hard neutrinos. This illustrates that a mass reconstruction procedure designed for the specific final state under consideration is mandatory to obtain the best possible sensitivity.

- Backgrounds. The backgrounds for $h \rightarrow \tau_{\text {had }} \tau_{\mu}$ and $h \rightarrow \tau_{\text {had }} \mu$ events are similar, but because of the different invariant mass reconstruction techniques, the reconstructed background spectra will typically be harder for a $h \rightarrow \tau_{\text {had }} \tau_{\mu}$ analysis, which assumes 
three neutrinos in the final state, than for a $h \rightarrow \tau_{\text {had }} \mu$ analysis which assumes only one. This implies, for instance, that the peak from the $Z \rightarrow \tau \tau$ background will appear at a $\tau_{\text {had }} \tau_{\mu}$ invariant mass around $90 \mathrm{GeV}$ in a search for $h \rightarrow \tau_{\text {had }} \tau_{\mu}$, but well below (and thus further away from the signal peak) in a dedicated $h \rightarrow \tau_{\text {had }} \mu$ analysis.

These considerations show that the LHC is potentially more sensitive to flavor violating $h \rightarrow \tau_{\text {had }} \mu$ decays than to the SM $h \rightarrow \tau \tau$ channel. We now discuss a possible strategy for a tailored $h \rightarrow \tau_{\text {had }} \mu$ analysis.

\subsection{A dedicated $h \rightarrow \tau \mu$ analysis}

We now investigate the potential of a dedicated $h \rightarrow \tau_{\text {had }} \mu$ analysis which follows closely the CMS search for $h \rightarrow \tau_{\text {had }} \tau_{\mu}$ [27]..$^{5}$ The most important difference to that analysis will be a different algorithm for reconstructing the $\tau \mu$ invariant mass. In particular, since the $\tau_{\text {had }} \mu$ final state contains only one neutrino (from the hadronic $\tau$ ), this mass reconstruction can always be done exactly (i.e. the neutrino momentum can be determined) up to a two-fold ambiguity.

An important background for $h \rightarrow \tau \mu$ is $Z+$ jets, where either the $Z$ decays into $\tau^{+} \tau^{-}$ and one of the $\tau$ 's decays further into a muon, or the $Z$ decays into $\mu^{+} \mu^{-}$and one of the jets fakes a $\tau$. Another important background is $W+$ jets, followed by $W \rightarrow \mu \nu_{\mu}$ and a jet faking a $\tau$. We neglect the small $t \bar{t}$ background, where a final state $\tau$ can come from a $W$ decay or be faked by a jet, and a muon can originate from a $W$ decay or from a leptonic $\tau$ decay. We also do not consider backgrounds from QCD multijet production because making reliable predictions for these events requires full detector simulations. Based on the CMS $h \rightarrow \tau_{\mu} \tau_{\text {had }}$ search [27] we expect them to be about as large as the $W+$ jets background in the invariant mass region around $125 \mathrm{GeV}$.

To simulate the parton-level signal and background events, we use MadGraph 5 v1.4.6 [57], with an extended version of the Higgs Effective Theory model to include flavor-violating Higgs interactions. We use Pythia 6.4 for parton showering and hadronization and Delphes 2.0.2 [60] as a fast detector simulation. We have compared the $\tau$ detection efficiency as well as the fake rate from QCD jets in Delphes 2.0.2 to the corresponding performance indicators of several CMS $\tau$ tagging algorithm. With a tagging efficiency of $\sim 0.2$ and a fake rate between $0.2 \%$ at low $p_{T}$ and $1 \%$ at $p_{T} \gtrsim 60 \mathrm{GeV}$, Delphes somewhat underestimates the performance of the CMS $\tau$ tagging algorithms [61]. (We have also studied $\tau$ tagging in PGS [58], but found it to be even farther away from what CMS can achieve.) To compensate for the imperfections in our treatment of $\tau$-tagging, and for other inaccuracies in our simulations, we normalize our background distributions to the expected event numbers from [27], table 2. We also normalize the $h \rightarrow \tau \mu$ signal using the same scaling factor as for the $\mathrm{SM} h \rightarrow \tau_{\text {had }} \tau_{\mu}$ events.

In the analysis we require exactly one muon with $p_{T}>17 \mathrm{GeV}$ and $|\eta|<2.1$ in the final state and exactly one jet tagged as a hadronic $\tau$ decay with $p_{T}>20 \mathrm{GeV}$ and $|\eta|<2.3$.

\footnotetext{
${ }^{5}$ For semileptonically decaying $\tau$, search strategies similar to the ones investigated in ref. [59] for flavor violating $Z \rightarrow \tau \mu$ decays could be used.
} 

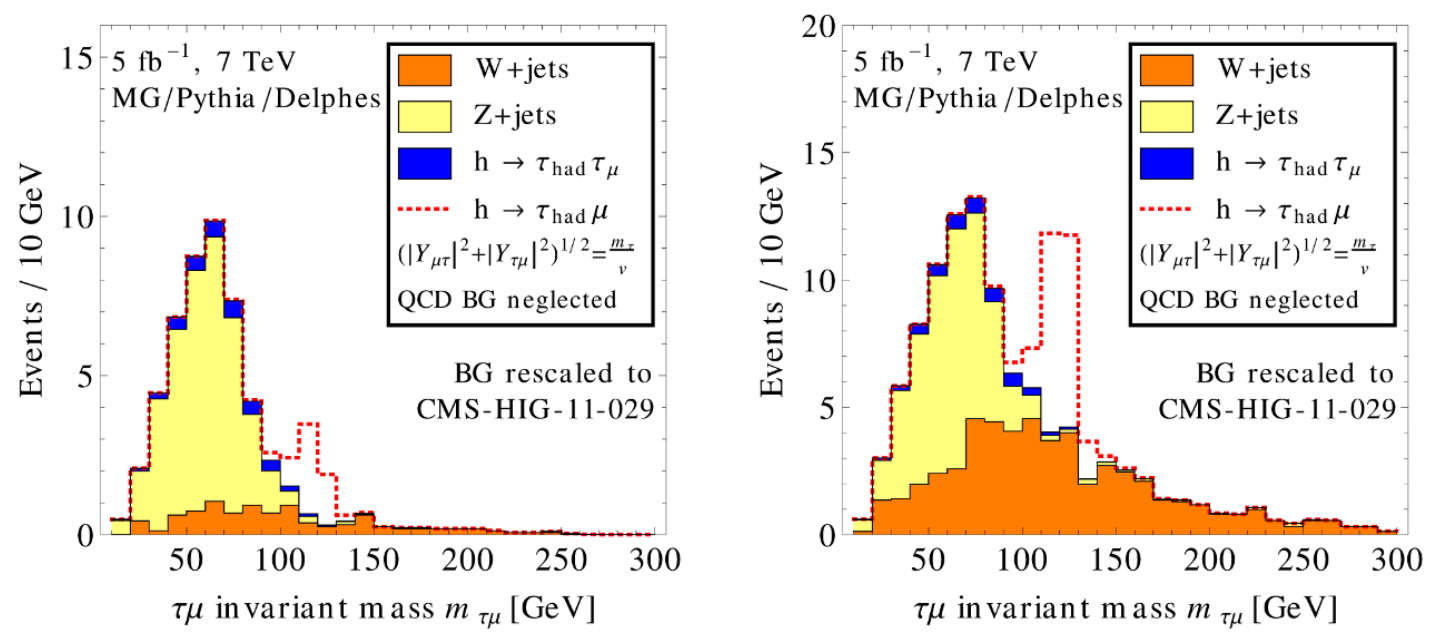

Figure 10. Signal and background rates for $h \rightarrow \tau \mu$ events in a CMS-like search (see text) as a function of the reconstructed $\mu-\tau$ invariant mass $m_{\tau \mu}$ for a vector boson fusion-enriched event sample. In the left panel the transverse mass cut $m_{T}\left(\mu, \mathbf{p}_{\text {miss }, T}\right)<40 \mathrm{GeV}$ is included, while in the right panel it is omitted. The QCD multijet background and the small $t \bar{t}$ background, are not included. The value chosen for $\sqrt{Y_{\tau \mu}^{2}+Y_{\mu \tau}^{2}}$ is well within the region allowed by other searches for flavor violation in the $\mu-\tau$ system (see section 3 ).

The muon and the $\tau$ are required to have opposite charge. In [27], it was found that the best signal-to-background ratio is achieved in the events where the Higgs boson was produced through vector boson fusion (VBF), and we confirm this in our own simulations. To enrich the data sample in VBF events, we consider only events with a pair of jets $j_{1}$, $j_{2}$ satisfying $|\Delta \eta|>4.0, \eta_{1} \eta_{2}<0, m_{j j}>400 \mathrm{GeV}$ and no other jets with $p_{T}>30 \mathrm{GeV}$ in the pseudorapidity region between the $j_{1}$ and $j_{2}$. Here, $\Delta \eta=\eta_{1}-\eta_{2}$ is the pseudorapidity difference between the two jets and $m_{j j}$ is the invariant mass of the jet pair. Non- $\tau$ jets are included in the analysis so long as their $p_{T}$ is above $30 \mathrm{GeV}$ and their pseudorapidity is $|\eta|<4$.7. In the CMS analysis [27], the transverse mass of the muon and the missing energy is restricted to be below $40 \mathrm{GeV}$ in order to suppress the $W+$ jets background. This works because in $W+$ jets events, the muon and the neutrino from $W \rightarrow \mu \nu_{\mu}$ tend to be more back-to-back than in $h \rightarrow \tau_{\mu} \tau_{\text {had }}$, where both $\tau$ 's contribute to the missing energy. In $h \rightarrow \tau_{\text {had }} \mu$, however, the muon and the missing energy also tend to be back-to-back, so that the $m_{T}\left(\mu, \mathbf{p}_{\text {miss }, T}\right)$ cut also removes a large fraction of signal events.

In light of this we show in figure 10 the expected signal and background rates for $h \rightarrow$ $\tau \mu$ as a function of the $\mu-\tau$ invariant mass $m_{\tau \mu}$ both with and without the transverse mass cut. In computing $m_{\tau \mu}$ for each event, we have used energy and momentum conservation to compute the $z$-component of the neutrino momentum $p_{\nu, z}$. There are two solutions to these equations, and we arbitrarily pick the smaller of the two. (We have checked that choosing the larger value for $p_{\nu, z}$ yields a very similar plot. This is related to the fact that $m_{\tau} \ll m_{h}$, so that the $\tau$ 's decay products are almost collinear.) As shown in the right panel of figure 10, dropping the transverse mass cut increases the $W$ plus jets background, but has the benefit of retaining more signal. The transverse mass distributions for signal 


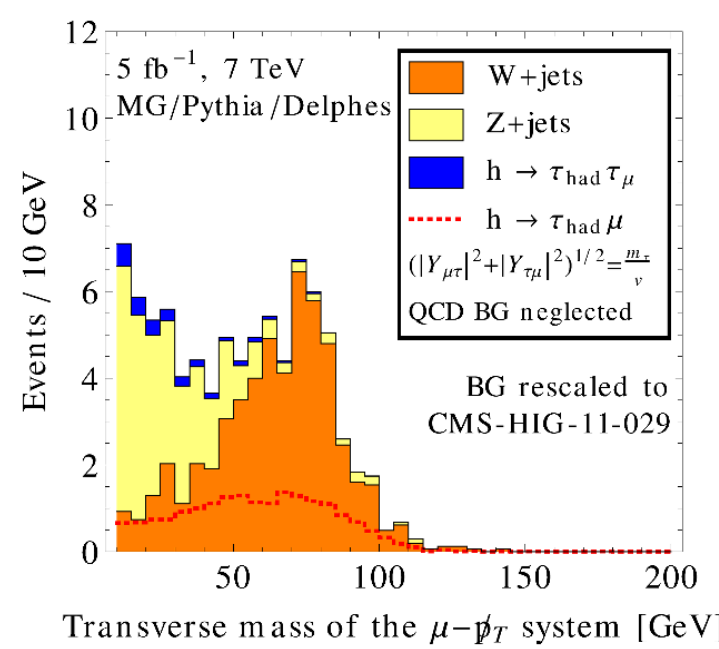

Figure 11. The transverse mass distribution of the muon-missing energy system for the backgrounds and for the $\tau_{\text {had }} \mu$ signal.

and background are shown in figure 11. Assuming the $W$ plus jets and QCD backgrounds can be controlled reasonably, relaxing this cut may be worthwhile.

In summary, figure 10 shows that for flavor violating Yukawa couplings well allowed by low energy precision measurements, a spectacular signal can be expected in a dedicated search at the LHC. Such a search would cut deeply into the allowed parameter space of the flavor violation Higgs to $\tau \mu$ couplings.

\section{Conclusions}

The LHC experiments have recently discovered a Higgs-like resonance with a mass around $125 \mathrm{GeV}$. In this paper we have examined the constraints on potential flavor violating couplings of this resonance, assuming it is indeed a scalar boson. In deriving the constraints we have assumed that that flavor changing neutral currents are dominated by the Higgs contributions, which may be thought of as a "simplified model" approach to flavor violation in light of the Higgs discovery. (In a complete model, cancellations between Higgs-induced flavor violation and flavor violation induced by other new physics is possible, but we do not pursue this possibility here.)

We have refined the indirect constraints on the flavor violating Yukawa couplings $Y_{i j}$ using results from rare decay searches, magnetic and electric dipole moment measurement, and the LHC. All constraints are summarized in tables 1 and 2 and in figures 6 and 8. We have compared the bounds to the loose naturalness criterion that the off-diagonal Yukawa couplings are not much bigger then the geometric mean of diagonal terms, $Y_{i j} \lesssim \sqrt{Y_{i i} Y_{j j}}$, and we have discussed to what extent the LHC can probe flavor violating decays of the form $h \rightarrow \bar{f}_{i} f_{j}$ (or in the case of flavor violating top-Higgs couplings the decay $t \rightarrow h f_{i}$ ).

We draw the following conclusions:

Natural Flavor Violation. The existing constraints involving only the first two generations of fermions, quarks or leptons, are strong enough that natural FV is already being 
probed by meson oscillations, $\mu \rightarrow e$ conversion and $\mu \rightarrow e \gamma$. This conclusion also holds for FV couplings to $b$ quarks. In contrast, the FV couplings involving $\tau$ leptons or top quarks are allowed to have natural size (unless there is a large hierarchy between $Y_{i j}$ and $Y_{j i}$ ). This means that they are potentially observable, either at the LHC or in future low energy experiments.

Opportunities for the LHC. The LHC has an opportunity to probe a large part of the allowed parameter space for $h \rightarrow \tau \mu$ and $h \rightarrow \tau e$ couplings. An LHC search in these channels would be very similar to the existing searches for $h \rightarrow \tau \tau$, and recasting the latter already gives the best bounds on the flavor violating Yukawa couplings $Y_{\tau e}, Y_{e \tau}$, $Y_{\tau \mu}$, and $Y_{\mu \tau}$ already now. A dedicated LHC search could improve the limits significantly. The reason why Higgs decays are very constraining is that the SM width of a $125 \mathrm{GeV}$ Higgs boson is very small, $\Gamma_{h} \simeq 4 \mathrm{MeV}$ [46], so that the flavor violating couplings of the Higgs can have a significant effect. Another illustration of the LHC's discovery potential for flavor violating Higgs couplings is that even the global fits of potential deviations in the dominant SM Higgs decay modes, $h \rightarrow W W, Z Z, b \bar{b}, \tau \tau, \gamma \gamma$, already give meaningful bounds on the FV Higgs decays. The results from these global fits are usually presented as bounds on the invisible decay width of the Higgs, but these bounds applies equally well to the sum of all the modes that have not been included in the fits. The constraint $\mathrm{BR}(h \rightarrow$ invisible $) \lesssim \mathcal{O}(70 \%)$ (at $95 \% \mathrm{CL}$, with modest theory assumptions [47, 62-64]) is comparable to the constraints on $\mathrm{BR}(h \rightarrow \tau \mu)$ and $\mathrm{BR}(h \rightarrow \tau e)$ from precision searches of FCNCs in the lepton sector.

Finally, flavor violating Higgs couplings involving the top quark are poorly constrained, and in fact, the LHC already provides the strongest limits on such couplings, see figure 8 and section 4 .

\section{Acknowledgments}

We thank Edward Boos, Zackaria Chacko, Ricky Fok, Graham Kribs, Ethan Neil, Takemichi Okui, and Ze'ev Surujon for valuable discussions, and LOT Polish Airlines for free internet access during a crucial phase of this project. This material is based upon work supported in part by the National Science Foundation under Grant No. PHYS-1066293 and the hospitality of the Aspen Center for Physics. JZ was supported in part by the U.S. National Science Foundation under CAREER Grant PHY1151392. Fermilab is operated by Fermi Research Alliance under contract DE-AC02-07CH11359 with the United States Department of Energy.

\section{A Further details on leptonic FCNCs}

In this appendix we collect detailed expressions for the FCNC processes $\tau \rightarrow \mu \gamma$ and $\mu \rightarrow e$ conversion in nuclei. 


\section{A.1 One loop expressions for $\tau \rightarrow \mu \gamma, \tau \rightarrow e \gamma, \mu \rightarrow e \gamma$}

The $\tau \rightarrow \mu \gamma$ effective Lagrangian is given in eq. (3.3). The Wilson coefficients $c_{L, R}$ are given by

$$
\begin{aligned}
& c_{L}=F\left(m_{\tau}, m_{\tau}, m_{\mu}, m_{h}, 0, Y\right)+F\left(m_{\tau}, m_{\mu}, m_{\mu}, m_{h}, 0, Y\right), \\
& c_{R}=F\left(m_{\tau}, m_{\tau}, m_{\mu}, m_{h}, 0, Y^{\dagger}\right)+F\left(m_{\tau}, m_{\mu}, m_{\mu}, m_{h}, 0, Y^{\dagger}\right),
\end{aligned}
$$

with the loop functions

$$
\begin{aligned}
F\left(m_{i}, m_{f}, m_{j}, m_{h}, q^{2}, Y\right)= & \frac{1}{4 m_{i}} \int_{0}^{1} d x d y d z \delta(1-x-y-z) \\
& \frac{x z m_{j} Y_{j f} Y_{i f}^{*}+y z m_{i} Y_{f j}^{*} Y_{f i}+(x+y) m_{f} Y_{f j}^{*} Y_{i f}^{*}}{z m_{h}^{2}-x z m_{j}^{2}-y z m_{i}^{2}+(x+y) m_{f}^{2}-x y q^{2}} .
\end{aligned}
$$

Here $m_{\mu}, m_{\tau}$ and $m_{h}$ are the muon, tau and Higgs masses, respectively, $q$ is the 4momentum of the photon, and $Y$ is the Yukawa coupling matrix. Note that $c_{L}$ and $c_{R}$ differ only by the replacement $Y_{i j} \leftrightarrow Y_{j i}^{*}$. The first terms in eqs. (A.1) and (A.2) arise from the first diagram in figure 1 (with a $\tau$ propagating in the loop), whereas the second terms arise from the second diagram (with a $\mu$ in the loop). Expanding in powers of $m_{\mu} / m_{\tau}$ and $m_{\tau} / m_{h}$ and keeping only the leading terms (so that only the first terms in (A.1), (A.2) contribute), the above expressions simplify to (3.5) if the diagonal Yukawa couplings are real. The simplified expressions for $\tau \rightarrow e \gamma$ and $\mu \rightarrow e \gamma$ (with a muon running in the loop) are obtained from (3.5) with trivial modifications, while the simplified expression for $\mu \rightarrow e \gamma$ with a $\tau$ running in the loop is given in eq. (3.8).

\section{A.2 Two loop expressions for $\tau \rightarrow \mu \gamma, \tau \rightarrow e \gamma$ and $\mu \rightarrow e \gamma$}

At two loops there are numerically important diagrams with top or $W$ running in the loop, attached to the Higgs. Here we translate the results of [36] into our notation and adapt them to the case of $\tau \rightarrow \mu \gamma$. The diagrams with top and photon in the loops (see figure 12 top left) contribute as

$$
\Delta c_{L}^{t \gamma}=-6 \kappa Q_{t}^{2} \frac{v}{m_{t}} Y_{\tau \mu}^{*}\left[\operatorname{Re}\left(Y_{t t}\right) f\left(z_{t h}\right)-i \operatorname{Im}\left(Y_{t t}\right) g\left(z_{t h}\right)\right],
$$

while the $W$-photon 2-loop contribution is

$$
\Delta c_{L}^{W \gamma}=\kappa Y_{\tau \mu}^{*}\left[3 f\left(z_{W h}\right)+5 g\left(z_{W h}\right)+\frac{3}{4} g\left(z_{W h}\right)+\frac{3}{4} h\left(z_{W h}\right)+\frac{f\left(z_{W h}\right)-g\left(z_{w h}\right)}{2 z_{W h}}\right] .
$$

Here we have already added the contributions from the would-be Goldstone bosons that get eaten by the $W$. The contributions to $\Delta c_{R}^{i}$ are obtained from the above by replacing $Y_{\tau \mu}^{*} \rightarrow Y_{\mu \tau}$ and $Y_{t t} \rightarrow Y_{t t}^{*}$. The loop functions are

$$
\begin{aligned}
& f(z)=\frac{1}{2} z \int_{0}^{1} d x \frac{1-2 x(1-x)}{x(1-x)-z} \log \frac{x(1-x)}{z}, \\
& g(z)=\frac{1}{2} z \int_{0}^{1} d x \frac{1}{x(1-x)-z} \log \frac{x(1-x)}{z}
\end{aligned}
$$



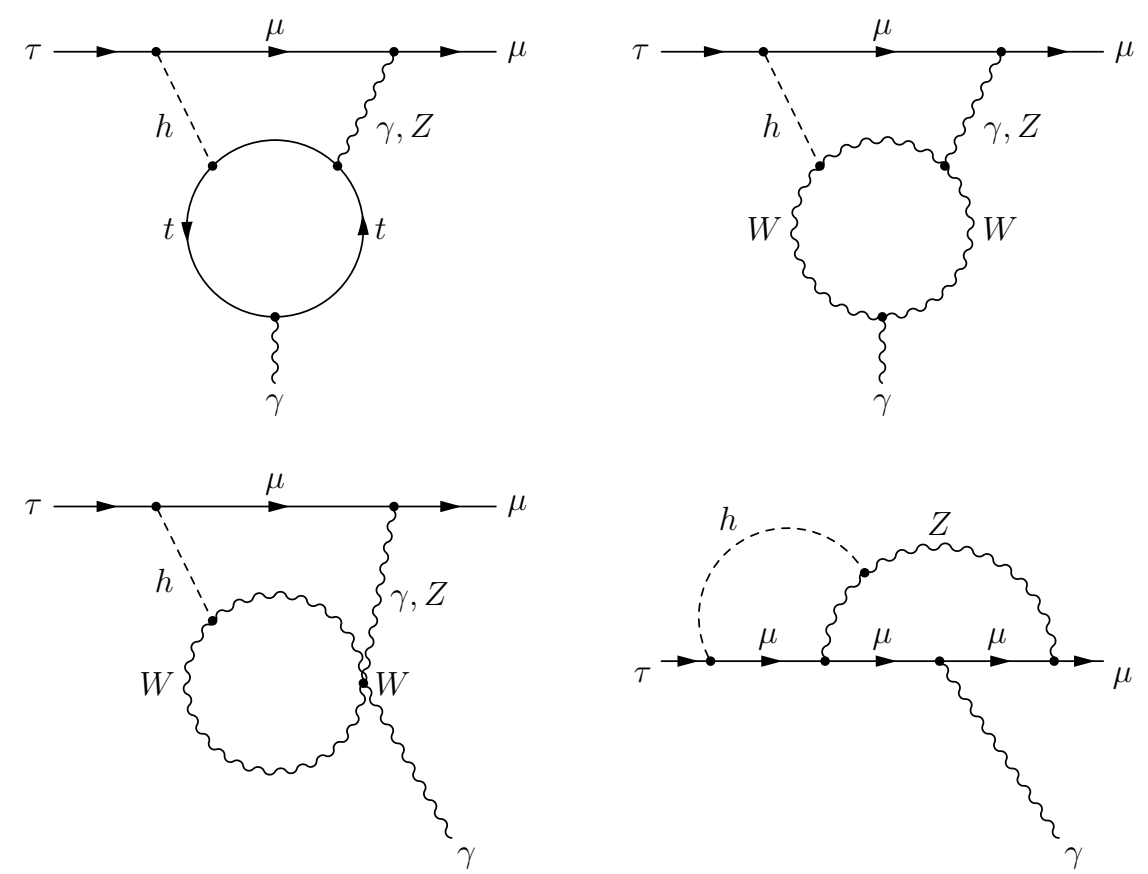

Figure 12. 2-loop diagrams contributing to $\tau \rightarrow \mu \gamma$.

$$
h(z)=z^{2} \frac{\partial}{\partial z}\left(\frac{g(z)}{z}\right)=\frac{z}{2} \int_{0}^{1} \frac{d x}{z-x(1-x)}\left[1+\frac{z}{z-x(1-x)} \log \frac{x(1-x)}{z}\right]
$$

the arguments are $z_{t h}=m_{t}^{2} / m_{H}^{2}, z_{W h}=m_{W}^{2} / m_{H}^{2}$, while the prefactor is

$$
\kappa=\frac{\alpha}{16 \pi} \frac{g^{2}}{m_{W}^{2}} \frac{v}{m_{\tau}}=\frac{\alpha}{2 \sqrt{2} \pi} G_{F} \frac{v}{m_{\tau}} .
$$

The contributions from the 2-loop diagrams with an internal $Z$ are smaller as they are suppressed by $1-4 s_{W}^{2} \simeq 0.08$. They are

$$
\begin{aligned}
\Delta c_{L}^{t Z}= & -6 \kappa Q_{t} \frac{\left(1-4 s_{W}^{2}\right)\left(1-4 Q_{t} s_{W}^{2}\right)}{16 s_{W}^{2} c_{W}^{2}} \frac{v}{m_{t}} Y_{\tau \mu}^{*} \times \\
& \times\left[\operatorname{Re}\left(Y_{t t}\right) \tilde{f}\left(z_{t h}, z_{t Z}\right)-i \operatorname{Im}\left(Y_{t t}\right) \tilde{g}\left(z_{t h}, z_{t Z}\right)\right], \\
\Delta c_{L}^{W Z}= & \kappa \frac{1-4 s_{W}^{2}}{4 s_{W}^{2}} Y_{\tau \mu}^{*}\left\{\frac{1}{2}\left(5-t_{W}^{2}\right) \tilde{f}\left(z_{t h}, z_{W Z}\right)+\frac{1}{2}\left(7-3 t_{W}^{2}\right) \tilde{g}\left(z_{t h}, z_{W Z}\right)\right. \\
& \left.+\frac{3}{4} g\left(z_{t h}\right)+\frac{3}{4} h\left(z_{t h}\right)+\frac{1}{4 z_{t h}}\left(1-t_{W}^{2}\right)\left[\tilde{f}\left(z_{t h}, z_{W Z}\right)-\tilde{g}\left(z_{t h}, z_{W Z}\right)\right]\right\},
\end{aligned}
$$

with $s_{W} \equiv \sin \theta_{W}, c_{W} \equiv \cos \theta_{W}, t_{W} \equiv \tan \theta_{W}, z_{t z} \equiv m_{t}^{2} / m_{Z}^{2}, z_{W Z} \equiv m_{W}^{2} / m_{Z}^{2}$ and the loop functions

$$
\tilde{f}(x, y)=\frac{y f(x)}{y-x}+\frac{x f(y)}{x-y}, \quad \tilde{g}(x, y)=\frac{y g(x)}{y-x}+\frac{x g(y)}{x-y} .
$$

The $\Delta c_{R}^{i}$ are obtained by replacing $Y_{\mu e}^{*} \rightarrow Y_{e \mu}$ and $Y_{t t} \rightarrow Y_{t t}^{*}$ in the above expressions. In addition there are also contributions called "set C" in [36]. An example for one of these 
diagrams is the last diagram in figure 12. Since the expressions for these diagrams are long we do not write them out explicitly. The "set C" contribution to $\Delta c_{L}$ is obtained from [36] by multiplying their eq. (20) by $-\kappa$ and replacing $\sum_{a} \cos \varphi_{a} \Delta_{e \mu}^{a} \rightarrow Y_{\tau \mu}^{*}$.

The $\tau \rightarrow e \gamma$ expressions are obtained by replacing $Y_{\tau \mu} \rightarrow Y_{\tau e}$ and $Y_{\mu \tau} \rightarrow Y_{e \tau}$ in the above expressions, while for $\mu \rightarrow e \gamma$ the replacements are $Y_{\tau \mu} \rightarrow Y_{\mu e}, Y_{\mu \tau} \rightarrow Y_{e \mu}$ and $m_{\tau} \rightarrow m_{\mu}$.

\section{A.3 Details on $\mu \rightarrow e$ conversion bounds}

The most general effective Lagrangian for $\mu \rightarrow e$ conversion in nuclei is [65]

$$
\begin{aligned}
\mathcal{L}= & c_{L} \frac{e}{8 \pi^{2}} m_{\mu}\left(\bar{e} \sigma^{\alpha \beta} P_{L} \mu\right) F_{\alpha \beta}-\frac{1}{2} \sum_{q}\left[g_{L S}^{q}\left(\bar{e} P_{R} \mu\right)(\bar{q} q)+g_{L P}^{q}\left(\bar{e} P_{R} \mu\right)\left(\bar{q} \gamma_{5} q\right)\right. \\
& \left.+g_{L V}^{q}\left(\bar{e} \gamma^{\mu} P_{L} \mu\right)\left(\bar{q} \gamma_{\mu} q\right)+g_{L A}^{q}\left(\bar{e} \gamma^{\mu} P_{L} \mu\right)\left(\bar{q} \gamma_{\mu} \gamma_{5} q\right)+\frac{1}{2} g_{L T}^{q}\left(\bar{e} \sigma^{\alpha \beta} P_{R} \mu\right)\left(\bar{q} \sigma_{\alpha \beta} q\right)\right]+L \leftrightarrow R .
\end{aligned}
$$

The above operators are generated by integrating out the Higgs in the diagrams shown in figure 5. The magnetic penguin Wilson coefficients $c_{L}$ and $c_{R}$ are (in analogy to eqs. (A.1) and (A.2))

$$
\begin{aligned}
& c_{L}=F\left(m_{\mu}, m_{\mu}, m_{e}, m_{h},-m_{\mu}^{2}, Y\right)+F\left(m_{\mu}, m_{e}, m_{e}, m_{h},-m_{\mu}^{2}, Y\right), \\
& c_{R}=F\left(m_{\mu}, m_{\mu}, m_{e}, m_{h},-m_{\mu}^{2}, Y^{\dagger}\right)+F\left(m_{\mu}, m_{e}, m_{e}, m_{h},-m_{\mu}^{2}, Y^{\dagger}\right),
\end{aligned}
$$

with the function $F\left(m_{i}, m_{f}, m_{j}, m_{h}, q^{2}, Y\right)$ given in eq. (A.3). Here we have set $q^{2} \simeq-m_{\mu}^{2}$ for the 4-momentum transfer to the nucleus, which is justified in the limit of an infinitely heavy nucleus. The above contributions are obtained from the last two diagrams in figure 5 with either a muon or an electron running in the loop. These diagrams also generate the quark vector current operators with (in general $\tilde{g}_{L V}^{(p)}=2 g_{L V}^{u}+g_{L V}^{d}$ and $\tilde{g}_{L V}^{(n)}=2 g_{L V}^{d}+g_{L V}^{u}$ )

$$
\begin{aligned}
\tilde{g}_{L V}^{(p)}=g_{L V}^{q} / Q_{q}= & -\frac{\alpha}{2 \pi q^{2}}\left[G\left(m_{\mu}, m_{\mu}, m_{e}, m_{h}, q^{2}, Y\right)-G\left(m_{\mu}, m_{\mu}, m_{e}, m_{h}, 0, Y\right)\right. \\
& \left.+G\left(m_{\mu}, m_{e}, m_{e}, m_{h}, q^{2}, Y\right)-G\left(m_{\mu}, m_{e}, m_{e}, m_{h}, 0, Y\right)\right] .
\end{aligned}
$$

Here, $Q_{q}$ is the charge of quark $q$, and $\tilde{g}_{R V}^{(p)}, g_{R V}^{q}$ are given by eq. (A.16) with the replacement $Y \rightarrow Y^{\dagger}$. The loop function in (A.16) is

$$
\begin{aligned}
G\left(m_{i}, m_{f}, m_{j}, m_{h}, q^{2}, Y\right)= & \int_{0}^{1} d x \int_{0}^{1-x} d y\left[Y_{j f} Y_{i f}^{*} \log \Delta-\frac{1}{\Delta} m_{i} m_{j} z^{2} Y_{f j}^{*} Y_{f i}\right. \\
& \left.-\frac{1}{\Delta}\left(m_{f} m_{j} z Y_{f j}^{*} Y_{i f}^{*}+m_{f} m_{i} z Y_{j f} Y_{f i}+\left[q^{2} x y+m_{f}^{2}\right] Y_{j f} Y_{i f}^{*}\right)\right]
\end{aligned}
$$

where we have defined $\Delta \equiv z m_{h}^{2}-x z m_{j}^{2}-y z m_{i}^{2}+(x+y) m_{f}^{2}-x y q^{2}$ and $z=1-x-y$. Note that we subtract the value of the one-loop vertex correction at $q^{2}=0$, which gets absorbed into the wave function and mass renormalizations. 
The coefficients $c_{L, R}$ also receive two loop contributions with either a top or a $W$ running in the loop. If we evaluate these contributions at $q^{2}=0$, so that the expressions are exactly the same as in the previous subsection on $\mu \rightarrow e \gamma$ decay, we find that both oneloop and two-loop contributions are subdominant (with the 2-loop contribution the larger of the two). They are roughly an order of magnitude smaller than the scalar contributions, so that the above approximations suffice for our purposes.

The scalar Wilson coefficients, generated from the first diagram in figure 5, are

$$
g_{L S}^{q}=-\frac{2}{m_{h}^{2}} Y_{\mu e}^{*} \operatorname{Re}\left(Y_{q q}\right), \quad g_{R S}^{q}=-\frac{2}{m_{h}^{2}} Y_{e \mu} \operatorname{Re}\left(Y_{q q}\right),
$$

while the remaining Wilson coefficients are zero, $g_{L P}^{q}=g_{R P}^{q}=g_{L A}^{q}=g_{R A}^{q}=g_{L T}^{q}=$ $g_{R T}^{q}=0$.

When computing the $\mu \rightarrow e$ conversion rate in nuclei care must be taken to account for the nuclear matrix elements $\langle N|\bar{q} q| N\rangle,\left\langle N\left|\bar{q} \gamma^{\mu} q\right| N\right\rangle$ and $\left\langle N\left|F^{\mu \nu}\right| N\right\rangle$ and for the overlap of the initial muon wave function and the final state electron wave function. We follow [65] and obtain

$$
\begin{aligned}
\Gamma(\mu \rightarrow e \text { conversion })= & \left|-\frac{e}{16 \pi^{2}} c_{L} D+\tilde{g}_{L S}^{(p)} S^{(p)}+\tilde{g}_{L S}^{(n)} S^{(n)}+\tilde{g}_{L V}^{(p)} V^{(p)}\right|^{2} \\
& +\left|-\frac{e}{16 \pi^{2}} c_{R} D+\tilde{g}_{R S}^{(p)} S^{(p)}+\tilde{g}_{R S}^{(n)} S^{(n)}+\tilde{g}_{R V}^{(p)} V^{(p)}\right|^{2} .
\end{aligned}
$$

The electromagnetic penguin and vector contributions are given in eqs. (A.14), (A.15), (A.16), while the scalar coefficients are

$$
\tilde{g}_{L S, R S}^{(p)}=\sum_{q} g_{L S, R S}^{q} \frac{m_{p}}{m_{q}} f^{(q, p)}, \quad \tilde{g}_{L S, R S}^{(n)}=\sum_{q} g_{L S, R S}^{q} \frac{m_{n}}{m_{q}} f^{(q, n)},
$$

and the sum runs over all quark flavors, $q=u, d, s, c, b, t$. Note that vector couplings to neutrons are absent due to the neutron's vanishing electric charge.

The nucleon matrix elements $f^{(q, p)} \equiv\left\langle p\left|m_{q} \bar{q} q\right| p\right\rangle / m_{p}$ are calculated according to [66], but using an updated value for the nucleon sigma term $\Sigma_{\pi N}=55 \mathrm{MeV}$ [67] (If the value of the nucleon sigma term is even smaller, as indicated by recent unquenched lattice results, our bounds would become weaker). The nucleon matrix elements are numerically

$$
f^{(u, p)}=f^{(d, n)}=0.024, \quad f^{(d, p)}=f^{(u, n)}=0.033, \quad f^{(s, p)}=f^{(s, n)}=0.25,
$$

while the contributions from the heavier quarks are

$$
f^{(c, p)}=f^{(b, p)}=f^{(t, p)}=\frac{2}{27}\left(1-\sum_{q=u, d, s} f^{(q, p)}\right) .
$$

with the same values for neutrons. In the above expressions, $m_{q}$ denotes a quark mass, $m_{p}$ is the proton mass, and $m_{n}$ is the neutron mass. The coefficients $D, V^{(p)}, S^{(p)}$, and $S^{(n)}$ are overlap integrals of the muon, electron and nuclear wave function. They are tabulated for various target materials in [65]. The best limits are obtained from bounds on $\mu \rightarrow e$ conversion on gold, $\Gamma(\mu \rightarrow e)_{\mathrm{Au}} / \Gamma_{\text {capture Au }}<7 \times 10^{-13}$ (90\% CL) [68], for which in units of $m_{\mu}^{5 / 2}$ the overlap integrals are $D=0.189, S^{(p)}=0.0614, V^{(p)}=0.0974, S^{(n)}=0.0918$, using the same distributions for neutrons and protons in the nucleus. For the SM capture rate, we use a value $\Gamma_{\text {capture } \mathrm{Au}}=13.07 \times 10^{6} \mathrm{~s}^{-1}$ in the calculation [65]. 
Open Access. This article is distributed under the terms of the Creative Commons Attribution License which permits any use, distribution and reproduction in any medium, provided the original author(s) and source are credited.

\section{References}

[1] ATLAS collaboration, Observation of an Excess of Events in the Search for the Standard Model Higgs boson with the ATLAS detector at the LHC, ATLAS-CONF-2012-093 (2012).

[2] CMS collaboration, Observation of a new boson with a mass near $125 \mathrm{GeV}$, CMS-PAS-HIG-12-020 (2012).

[3] ATLAS collaboration, Observation of a new particle in the search for the Standard Model Higgs boson with the ATLAS detector at the LHC, Phys. Lett. B 716 (2012) 1 [arXiv: 1207.7214] [INSPIRE].

[4] CMS collaboration, Observation of a new boson at a mass of $125 \mathrm{GeV}$ with the CMS experiment at the LHC, Phys. Lett. B 716 (2012) 30 [arXiv:1207.7235] [INSPIRE].

[5] CDF, D0 collaborations, T. Aaltonen et al., Evidence for a particle produced in association with weak bosons and decaying to a bottom-antibottom quark pair in Higgs boson searches at the Tevatron, Phys. Rev. Lett. 109 (2012) 071804 [arXiv:1207.6436] [INSPIRE].

[6] J. Bjorken and S. Weinberg, A Mechanism for Nonconservation of Muon Number, Phys. Rev. Lett. 38 (1977) 622 [INSPIRE].

[7] B. McWilliams and L.-F. Li, Virtual effects of Higgs particles, Nucl. Phys. B 179 (1981) 62 [INSPIRE].

[8] O.U. Shanker, Flavor Violation, Scalar Particles and Leptoquarks, Nucl. Phys. B 206 (1982) 253 [INSPIRE].

[9] S.M. Barr and A. Zee, Electric dipole moment of the electron and of the neutron, Phys. Rev. Lett. 65 (1990) 21 [Erratum ibid. 65 (1990) 2920] [INSPIRE].

[10] K. Babu and S. Nandi, Natural fermion mass hierarchy and new signals for the Higgs boson, Phys. Rev. D 62 (2000) 033002 [hep-ph/9907213] [INSPIRE].

[11] J.L. Diaz-Cruz and J. Toscano, Lepton flavor violating decays of Higgs bosons beyond the standard model, Phys. Rev. D 62 (2000) 116005 [hep-ph/9910233] [INSPIRE].

[12] T. Han and D. Marfatia, $h \rightarrow \mu \tau$ at hadron colliders, Phys. Rev. Lett. 86 (2001) 1442 [hep-ph/0008141] [INSPIRE].

[13] M. Blanke, A.J. Buras, B. Duling, S. Gori and A. Weiler, $\Delta F=2$ Observables and Fine-Tuning in a Warped Extra Dimension with Custodial Protection, JHEP 03 (2009) 001 [arXiv:0809.1073] [INSPIRE].

[14] S. Casagrande, F. Goertz, U. Haisch, M. Neubert and T. Pfoh, Flavor Physics in the Randall-Sundrum Model: I. Theoretical Setup and Electroweak Precision Tests, JHEP 10 (2008) 094 [arXiv: 0807. 4937] [INSPIRE].

[15] G.F. Giudice and O. Lebedev, Higgs-dependent Yukawa couplings, Phys. Lett. B 665 (2008) 79 [arXiv: 0804.1753] [INSPIRE].

[16] J. Aguilar-Saavedra, A Minimal set of top-Higgs anomalous couplings, Nucl. Phys. B 821 (2009) 215 [arXiv:0904.2387] [INSPIRE]. 
[17] M.E. Albrecht, M. Blanke, A.J. Buras, B. Duling and K. Gemmler, Electroweak and Flavour Structure of a Warped Extra Dimension with Custodial Protection, JHEP 09 (2009) 064 [arXiv:0903.2415] [INSPIRE].

[18] A.J. Buras, B. Duling and S. Gori, The Impact of Kaluza-Klein Fermions on Standard Model Fermion Couplings in a RS Model with Custodial Protection, JHEP 09 (2009) 076 [arXiv:0905.2318] [INSPIRE].

[19] K. Agashe and R. Contino, Composite Higgs-Mediated FCNC, Phys. Rev. D 80 (2009) 075016 [arXiv: 0906.1542] [inSPIRE].

[20] A. Goudelis, O. Lebedev and J.-h. Park, Higgs-induced lepton flavor violation, Phys. Lett. B 707 (2012) 369 [arXiv:1111.1715] [INSPIRE].

[21] A. Arhrib, Y. Cheng and O.C. Kong, Higgs to $\mu^{\mp} \tau^{ \pm}$Decay in Supersymmetry without R-parity, Europhys. Lett. 101 (2013) 31003 [arXiv:1208.4669] [INSPIRE].

[22] D. McKeen, M. Pospelov and A. Ritz, Modified Higgs branching ratios versus CP and lepton flavor violation, Phys. Rev. D 86 (2012) 113004 [arXiv:1208.4597] [INSPIRE].

[23] A. Azatov, M. Toharia and L. Zhu, Higgs Mediated FCNC's in Warped Extra Dimensions, Phys. Rev. D 80 (2009) 035016 [arXiv:0906.1990] [inSPIRE].

[24] G. Blankenburg, J. Ellis and G. Isidori, Flavour-Changing Decays of a $125 \mathrm{GeV}$ Higgs-like Particle, Phys. Lett. B 712 (2012) 386 [arXiv:1202.5704] [InSPIRE].

[25] S. Kanemura, T. Ota and K. Tsumura, Lepton flavor violation in Higgs boson decays under the rare tau decay results, Phys. Rev. D 73 (2006) 016006 [hep-ph/0505191] [INSPIRE].

[26] S. Davidson and G.J. Grenier, Lepton flavour violating Higgs and $\tau \rightarrow \mu \gamma$, Phys. Rev. D 81 (2010) 095016 [arXiv: 1001.0434] [INSPIRE].

[27] CMS collaboration, Search for neutral Higgs bosons decaying to $\tau$ pairs in pp collisions at $\sqrt{s}=7$ TeV, Phys. Lett. B 713 (2012) 68 [arXiv:1202.4083] [INSPIRE].

[28] H. Ishimori et al., Non-Abelian Discrete Symmetries in Particle Physics, Prog. Theor. Phys. Suppl. 183 (2010) 1 [arXiv: 1003.3552] [INSPIRE].

[29] G. Perez and L. Randall, Natural Neutrino Masses and Mixings from Warped Geometry, JHEP 01 (2009) 077 [arXiv: 0805.4652] [INSPIRE].

[30] BABAR collaboration, J. Lees et al., Evidence for an excess of $\bar{B} \rightarrow D^{(*)} \tau^{-} \bar{\nu}_{\tau}$ decays, Phys. Rev. Lett. 109 (2012) 101802 [arXiv:1205.5442] [INSPIRE].

[31] S. Fajfer, J.F. Kamenik, I. Nisandzic and J. Zupan, Implications of Lepton Flavor Universality Violations in B Decays, Phys. Rev. Lett. 109 (2012) 161801 [arXiv:1206.1872] [INSPIRE].

[32] W. Buchmüller and D. Wyler, Effective Lagrangian Analysis of New Interactions and Flavor Conservation, Nucl. Phys. B 268 (1986) 621 [InSPIRE].

[33] F. del Aguila, M. Pérez-Victoria and J. Santiago, Effective description of quark mixing, Phys. Lett. B 492 (2000) 98 [hep-ph/0007160] [INSPIRE].

[34] F. del Aguila, M. Pérez-Victoria and J. Santiago, Observable contributions of new exotic quarks to quark mixing, JHEP 09 (2000) 011 [hep-ph/0007316] [INSPIRE].

[35] T. Cheng and M. Sher, Mass Matrix Ansatz and Flavor Nonconservation in Models with Multiple Higgs Doublets, Phys. Rev. D 35 (1987) 3484 [INSPIRE]. 
[36] D. Chang, W. Hou and W.-Y. Keung, Two loop contributions of flavor changing neutral Higgs bosons to $\mu \rightarrow e \gamma$, Phys. Rev. D 48 (1993) 217 [hep-ph/9302267] [InSPIRE].

[37] Particle Data Group collaboration, J. Beringer et al., Review of Particle Physics (RPP), Phys. Rev. D 86 (2012) 010001 [inSPIRE].

[38] Particle Data Group collaboration, K. Nakamura et al., Review of particle physics, J. Phys. G 37 (2010) 075021 [inSPIRE].

[39] SINDRUM collaboration, U. Bellgardt et al., Search for the Decay $\mu^{+} \rightarrow e^{+} e^{+} e^{-}$, Nucl. Phys. B 299 (1988) 1 [INSPIRE].

[40] K. Hayasaka et al., Search for Lepton Flavor Violating Tau Decays into Three Leptons with 719 Million Produced $\tau^{+} \tau^{-}$Pairs, Phys. Lett. B 687 (2010) 139 [arXiv:1001.3221] [INSPIRE].

[41] L. Willmann et al., New bounds from searching for muonium to anti-muonium conversion, Phys. Rev. Lett. 82 (1999) 49 [hep-ex/9807011] [INSPIRE].

[42] T. Clark and S. Love, Muonium - anti-muonium oscillations and massive Majorana neutrinos, Mod. Phys. Lett. A 19 (2004) 297 [hep-ph/0307264] [INSPIRE].

[43] Muon G-2 collaboration, G. Bennett et al., Final Report of the Muon E821 Anomalous Magnetic Moment Measurement at BNL, Phys. Rev. D 73 (2006) 072003 [hep-ex/0602035] [INSPIRE].

[44] ATLAS collaboration, Search for Neutral MSSM Higgs bosons in $\sqrt{s}=7 \mathrm{TeV}$ pp collisions at ATLAS, ATLAS-CONF-2012-094 (2012).

[45] AlePh, DelPhi, L3, OPAL, LEP Electroweak Working Group collaborations, J. Alcaraz et al., A Combination of preliminary electroweak measurements and constraints on the standard model, hep-ex/0612034 [INSPIRE].

[46] S. Dittmaier et al., Handbook of LHC Higgs Cross sections: 2. Differential Distributions, arXiv:1201.3084 [INSPIRE].

[47] D. Carmi, A. Falkowski, E. Kuflik, T. Volansky and J. Zupan, Higgs After the Discovery: a Status Report, JHEP 10 (2012) 196 [arXiv:1207.1718] [INSPIRE].

[48] UTFit collaboration, M. Bona et al., Model-independent constraints on $\Delta F=2$ operators and the scale of new physics, JHEP 03 (2008) 049 [arXiv:0707.0636] [INSPIRE].

[49] D0 collaboration, V.M. Abazov et al., Search for flavor changing neutral currents via quark-gluon couplings in single top quark production using $2.3 \mathrm{fb}^{-1}$ of p $\bar{p}$ collisions, Phys. Lett. B 693 (2010) 81 [arXiv:1006.3575] [INSPIRE].

[50] N. Craig et al., Searching for $t \rightarrow$ ch with Multi-Leptons, Phys. Rev. D 86 (2012) 075002 [arXiv: 1207.6794] [INSPIRE].

[51] CDF collaboration, T. Aaltonen et al., Search for top-quark production via flavor-changing neutral currents in $W+1$ jet events at CDF, Phys. Rev. Lett. 102 (2009) 151801 [arXiv: 0812.3400] [INSPIRE].

[52] ATLAS collaboration, Search for FCNC single top-quark production at $\sqrt{s}=7 \mathrm{TeV}$ with the ATLAS detector, Phys. Lett. B 712 (2012) 351 [arXiv:1203.0529] [INSPIRE].

[53] CMS collaboration, Search for flavor changing neutral currents in top quark decays in pp collisions at 7 TeV, Phys. Lett. B 718 (2013) 1252 [arXiv:1208.0957] [INSPIRE]. 
[54] M. Pospelov and A. Ritz, Electric dipole moments as probes of new physics, Annals Phys. 318 (2005) 119 [hep-ph/0504231] [INSPIRE].

[55] ATLAS collaboration, Search for the Standard Model Higgs boson in the $H$ to $\tau^{+} \tau^{-}$decay mode in $\sqrt{s}=7 \mathrm{TeV}$ pp collisions with ATLAS, JHEP 09 (2012) 070 [arXiv:1206.5971] [INSPIRE].

[56] A. Elagin, P. Murat, A. Pranko and A. Safonov, A New Mass Reconstruction Technique for Resonances Decaying to $\tau \tau$, Nucl. Instrum. Meth. A 654 (2011) 481 [arXiv:1012.4686] [INSPIRE].

[57] J. Alwall, M. Herquet, F. Maltoni, O. Mattelaer and T. Stelzer, MadGraph 5: going Beyond, JHEP 06 (2011) 128 [arXiv:1106.0522] [INSPIRE].

[58] J. Conway et al., PGS - Pretty Good Simulation, http://physics.ucdavis.edu/ ${ }^{\sim}$ conway/research/software/pgs/pgs4-general.htm, (2009).

[59] S. Davidson, S. Lacroix and P. Verdier, LHC sensitivity to lepton flavour violating Z boson decays, JHEP 09 (2012) 092 [arXiv: 1207.4894] [INSPIRE].

[60] S. Ovyn, X. Rouby and V. Lemaitre, DELPHES, a framework for fast simulation of a generic collider experiment, arXiv:0903.2225 [INSPIRE].

[61] CMS collaboration, Tau identification in CMS, CMS-PAS-TAU-11-001.

[62] J.R. Espinosa, M. Muhlleitner, C. Grojean and M. Trott, Probing for Invisible Higgs Decays with Global Fits, JHEP 09 (2012) 126 [arXiv:1205.6790] [INSPIRE].

[63] A. Djouadi, A. Falkowski, Y. Mambrini and J. Quevillon, Direct Detection of Higgs-Portal Dark Matter at the LHC, arXiv:1205.3169 [INSPIRE].

[64] P.P. Giardino, K. Kannike, M. Raidal and A. Strumia, Is the resonance at $125 \mathrm{GeV}$ the Higgs boson?, Phys. Lett. B 718 (2012) 469 [arXiv:1207.1347] [INSPIRE].

[65] R. Kitano, M. Koike and Y. Okada, Detailed calculation of lepton flavor violating muon electron conversion rate for various nuclei, Phys. Rev. D 66 (2002) 096002 [Erratum ibid. D 76 (2007) 059902] [hep-ph/0203110] [INSPIRE].

[66] J.R. Ellis, K.A. Olive and C. Savage, Hadronic Uncertainties in the Elastic Scattering of Supersymmetric Dark Matter, Phys. Rev. D 77 (2008) 065026 [arXiv:0801.3656] [INSPIRE].

[67] R.D. Young and A.W. Thomas, Recent results on nucleon sigma terms in lattice QCD, Nucl. Phys. A 844 (2010) 266C-271C [arXiv:0911.1757] [InSPIRE].

[68] SINDRUM II collaboration, W.H. Bertl et al., A Search for muon to electron conversion in muonic gold, Eur. Phys. J. C 47 (2006) 337 [inSPIRE]. 